\title{
Spike-and-Wave Discharges Are Not Pathological Sleep Spindles, Network-Level Aspects of Age-Dependent Absence Seizure Development in Rats
}

\author{
[Dábor Kozák, ${ }^{1}$ (1)Tamás Földi, ${ }^{1}$ and (1)Antal Berényi ${ }^{1,2}$
}

https://doi.org/10.1523/ENEURO.0253-19.2019

${ }^{1}$ MTA-SZTE 'Momentum' Oscillatory Neuronal Networks Research Group, Department of Physiology, Interdisciplinary Excellence Centre, University of Szeged, Szeged H-6720, Hungary and ${ }^{2}$ Neuroscience Institute, New York University, New York City, NY 10016

\begin{abstract}
Spike-and-wave discharges (SWDs) of absence epilepsy are considered as pathologic alterations of sleep spindles; however, their network-level relationship has never been convincingly revealed. In order to observe the development and generalization of the thalamocortical SWDs and the concomitant alterations of sleep related oscillations, we performed local field potential (LFP) and single unit recordings in rats for three months during their maturation. We found that while SWDs and spindles look similar in young, they become different with maturation and shift to appear in different brain states. Thus, despite being generated by the same network, they are likely two distinct manifestations of the thalamocortical activity. We show that while spindles are already mainly global oscillations, SWDs appear mainly only focally in young. They become capable to generalize later with maturation, when the out-of-focus brain regions develop a decreased inhibitory/excitatory balance. These results suggest that a hyperexcitable focus is not sufficient alone to drive generalized absence seizures. Importantly, we also found the gradual age dependent disappearance of sleep spindles coinciding with the simultaneous gradual emergence of spike and waves, which both could be reversed by the proper dosing of ethosuximide (ETX). Based on these observations we conclude that the absence seizure development might be a multi-step process, which might involve the functional impairment of cortical interneurons and network-level changes that negatively affect sleep quality.
\end{abstract}

Key words: absence seizure; maturation; rat; sleep spindle; spike and wave; thalamocortical

\section{Significance Statement}

Absence seizure development parallels with the deterioration of slow-wave sleep (SWS), which might be reversible with ethosuximide (ETX) in a narrow therapeutic range, but not with early electrical seizure abruption. Thus, by proper dosing, spindles can be pharmaceutically recovered while epileptic activity is suppressed and absence related sleep and learning disturbances may be counterbalanced. We further found that despite the characteristic high voltage rhythmic global ictal EEG patterns the absence seizures are not accompanied with widespread phase locking of neuronal activities.

\section{Introduction}

Absence epilepsy is the most common epilepsy of childhood (Jallon et al., 2001). Spontaneous absence seizures in both humans and genetic rodent models are

Received June 29, 2019; accepted December 11, 2019; First published December 18, 2019.

A.B. is a shareholder in Amplipex Llc and Neunos Ltd., Szeged, Hungary, which manufacture biosignal amplifiers and neurostimulator devices. All other authors declare no competing financial interests. hypothesized to emerge from a hyperexcitable cortical focus (Holmes et al., 2004; Meeren et al., 2005), which generalizes involving the thalamocortical circuitry, and results in sudden loss of consciousness, behavioral arrest 
and a typical EEG pattern of spike-and-wave discharges (SWDs; Crunelli and Leresche, 2002; Blumenfeld, 2005). Importantly, the interplay between the cortex and the thalamus also gives rise to major physiologic oscillations such as spindles and $\delta$ waves during sleep (Steriade et al., 1993). Although these physiologic and pathologic oscillations can coexist in the same circuitry (Steriade et al., 1986; McCormick and Bal, 1997; Sanchez-Vives and McCormick, 2000; Timofeev and Steriade, 2004), very little is known about how the emergence of seizures influence the healthy sleep-related oscillations (Pinault et al., 2001; Beenhakker and Huguenard, 2009). To our knowledge, no study has investigated yet the evolution of the physiologic thalamocortical oscillations in relation to the emerging seizure activity.

Moreover, while numerous studies have provided important insights into the intraseizure and periseizure dynamics of thalamus and cortex, most of these works limited their scope to the investigation of the putative cortical focus and their thalamic peers (Steriade and Contreras, 1995; Pinault et al., 1998; Polack et al., 2007; McCafferty et al., 2018). Thus, it is largely unexplored how local ictal activity of the putative focus can evolve into fully developed, global absence seizures. Furthermore, the contribution of the out-of-focus cortical areas to the seizure evolution is also understudied. However, these questions are of a particular interest, as a recent study suggest that frequent seizures in absence epilepsy might alter the cortical microcircuitry in the seizure initiation zone (Studer et al., 2019). Thus, as fully developed absence epilepsy is a global phenomenon, it is reasonable to consider and investigate whether the out-of-focus cortical areas undergo changes due to the abundant seizures invading the whole cortex (Kozak, 2019).

To address these issues, we performed a longitudinal study in Long-Evans rats using multisite recording electrodes in multiple cortical areas to determine the evolution of spontaneous seizures and the related cooccurring alterations of sleep architecture. The evolution of absence seizures spans months in Long-Evans rats that capacitates this strain to be an ideal model to investigate how the gradual seizure emergence impacts other oscillations of the thalamocortical circuitry parallel to the progression of the epileptic condition. Additionally, to causally investigate the cortical mechanisms of SWD generalization and to understand how maturation influences seizure susceptibility, we also recorded spiking activity in juvenile (i.e., 2.5-4 months old) and mature (i.e., five to seven months

Innovation Office, Hungary; and the Ministry of Human Capacities, Hungary, Grant 20391-3/2018/FEKUSTRAT (to A.B.). G.K was supported by the Ministry of Human Capacities UNKP-18-3 New National Excellence Program of the Ministry of Human Capacities Grant UNKP-18-3-III-SZTE-16.

Acknowledgements: We thank Magor Lo"rincz, Antonio Fernández-Ruiz, Azahara Oliva, and Yuichi Takeuchi for insightful comments.

Correspondence should be addressed to Antal Berényi at drberenyi@gmail.com.

https://doi.org/10.1523/ENEURO.0253-19.2019

Copyright (C) 2020 Kozák et al.

This is an open-access article distributed under the terms of the Creative Commons Attribution 4.0 International license, which permits unrestricted use, distribution and reproduction in any medium provided that the original work is properly attributed. old) rats from out of focus cortical sites during spontaneous and electrically induced seizures employing highdensity silicone electrodes.

Here, we show that on the long-term, seizure evolution impacts the occurrence of sleep spindles, which is reversible with pharmacological treatment. Furthermore, we found that in contrast to the general belief, the majority of the cortical neurons are in silence during individual SWDs of the absence seizures; most cortical interneurons are suppressed, and pyramidal cells do not increase their firing rate either. Thus, absence seizures in the cortex might be rather related to a shifted inhibitory/excitatory balance than an increased, hypersynchronous discharge rate of the cortical units.

\section{Materials and Methods}

\section{Animals}

All experiments were performed in accordance with European Union guidelines (2003/65/CE) and the National Institutes of Health Guidelines for the Care and Use of Animals for Experimental Procedures. The experimental protocols were approved by the Ethical Committee for Animal Research at the Albert Szent-Györgyi Medical and Pharmaceutical Center of the University of Szeged (XIV/ 218/2016). Thirteen male Long-Evans rats were used in the present study.

\section{Surgery}

The animals (male Long-Evans rats) were operated under isoflurane anesthesia. The animals for longitudinal observations ( $n=5$, three months old), animals for pharmacological experiments ( $n=3$, five to seven months) and animals for transcranial electrical stimulation experiments ( $n=6$, five to seven months) were implanted with intracortical recording electrode triplets (interwire spacing, $0.4 \mathrm{~mm}$ ), which targeted the frontal and parietal cortical areas of both hemispheres and unilaterally the CA1 subfield of the hippocampus, as already reported elsewhere in details (Kozák and Berényi, 2017). For transcranial stimulation experiments, additional stimulation electrodes were implanted over the temporal bone bilaterally (Kozák and Berényi, 2017; Kozák et al., 2018). Unit recordings and intracortical stimulation experiments were performed in rats divided into two age groups: juvenile (2.5-4 months, $n=5$ ) and mature (five to seven months, $n=4$ ) animals. These age groups are referred to as "juvenile" and "mature" animals, throughout the manuscript. Rats were implanted with tripolar electrodes, which targeted the frontal and parietal cortical areas of the left hemisphere and unilaterally the CA1 subfield of the hippocampus and a silicone probe (Neuronexus, Poly2, 32 channels) with a custom-built microdrive (Vandecasteele et al., 2012) allowing for the vertical adjustment during targeting the prefrontal cortex (AP: $+2.7 \mathrm{~mm}$ from bregma; ML: +1.5 $\mathrm{mm}$, angled at $10^{\circ}$ from the sagittal plane) or motor cortex (AP: $-2 \mathrm{~mm}$ from bregma, ML: $+1.5 \mathrm{~mm}$ in the sagittal plane) of the right hemisphere. A custom-built bipolar electrode consisting of two insulated tungsten wires, each peeled at the tip for $200 \mu \mathrm{m}$ (interwire spacing, $0.5 \mathrm{~mm}$ ) was implanted in the left neocortex (AP: $+2 \mathrm{~mm}$; ML: -2 $\mathrm{mm}$; DV: $-1.5 \mathrm{~mm}$ from the dura (motor area; Maingret 
et al., 2016). Miniature stainless-steel screws (serving as reference and ground) were implanted above the cerebellum. A copper mesh (serving as a Faraday cage) was built around the probes and enforced with dental cement.

\section{Electrophysiological recordings and stimulation}

The rats were housed in the recording room individually in plastic cages, the walls were made of clear Plexiglas and food and water were given ad libitum. All recording sessions took place in the same room in 12/12 $\mathrm{h}$ light/dark cycles. After recovery from the surgery (minimum $3 \mathrm{~d}$ ), the maturing rats were connected to the recording system and were observed for three months continuously in their home cage, as reported elsewhere already (Kozák and Berényi, 2017). The neuronal signals were preamplified, amplified (total gain 400×), multiplexed on head and stored after digitalization at $20-\mathrm{kHz}$ sampling rate per channel (KJE1001, Amplipex).

To investigate the effect of antiepileptic treatment on sleep spindles, animals $(n=3)$ received an intraperitoneal injection of saline (control day) then on the following day an intraperitoneal injection of ethosuximide (ETX; treatment day, $100 \mathrm{mg} / \mathrm{kg}$ body weight; Richards et al., 2003). Injections were given at 8 a.m. and animals were monitored for 12 $\mathrm{h}$ in daylight. These control-treatment sessions were repeated three times, interleaved with 2-d-long rests between the consecutive sessions to ensure the elimination of ETX between measurements (Bachmann et al., 1988).

To investigate the effect of closed-loop transcranial electrical disruption of seizure activity on sleep patterns, animals implanted with transcranial stimulation and intracortical recording electrodes were connected to a custom programmed unsupervised seizure detection and intervention system (RX-8, Tucker-Davis Technologies), reported elsewhere in details (Kozák and Berényi, 2017). Each detected seizure triggered a charge neutral, triphasic single-pulse (100 ms) stimulation (STG4008; Multi Channel Systems) through the bitemporal stimulation electrodes. The closedloop $(n=3)$ and open-loop $(n=3)$ stimulated animals received treatment in an alternating fashion: each nonstimulated day (control) was followed by a stimulated day (treatment). The stimulation of each open-loop treated animal was driven by the seizure activity of a randomly chosen closed-loop treated animal.

In intracortical stimulation experiments the optimal stimulation voltage was determined in advance for each animal as the minimum voltage (7-15 V) necessary to reliably induce $\delta$ waves during NREM sleep. During stimulation a monophasic single-pulse $(0.1 \mathrm{~ms})$ was delivered by an isolated stimulator generator (STG4008; Multi Channel Systems) to the deep layers of the motor cortex (anode: $-1.5 \mathrm{~mm}$ from the dura, cathode: $-1.0 \mathrm{~mm}$ from the dura). Animals were recorded in their home cage both in control and stimulation sessions. In stimulation sessions intracortical pulses were delivered every $10 \mathrm{~s}$, with no respect to the behavioral states.

\section{Data analysis}

All analyses were conducted off-line. For aging experiment, a random day recording of each week was included. The raw wide-band signal was low-pass filtered and down-sampled to $1250 \mathrm{~Hz}$ to generate local field potential (LFP). SWD episodes were detected from the LFP signals recorded in the somatosensory cortex. Forth order Butterworth filter was applied to the LFP (in ranges of 8-12 and 30-200 Hz). Concurrent threshold-crossings in both range were considered as SWD events (Kozák and Berényi, 2017). Consecutive SWD episodes were merged if they followed each other within $1 \mathrm{~s}$. Events shorter than $1 \mathrm{~s}$ were excluded from the analysis. For $\delta$ wave and sleep spindle detections, we only used non- $\theta$ epochs. Non- $\theta$ epochs were detected automatically using the ratio of the power in $\theta$ band $(5-11 \mathrm{~Hz})$ to the power of nearby bands (1-4 and 12-14 Hz) of hippocampal LFP (Sirota et al., 2008). Threshold was manually adjusted for all animals. To detect global $\delta$ waves, the LFPs recorded bilaterally in the somatosensory were filtered $(0-6 \mathrm{~Hz})$ and Z-scored. Zero-crossings of the first temporal derivatives were calculated. Consecutive upward-downward-upward zerocrossings within a temporal window of 150 and $500 \mathrm{~ms}$ were considered as putative $\delta$ events. $\delta$ Waves corresponded to epochs where Z-score exceeded 2 at the peak, or exceeded 1 and fell below -1.5 at the end of the event bilaterally (Maingret et al., 2016). $\delta$ Waves were only used to identify slow-wave sleep (SWS) sessions and to approximate the depth of SWS (Gervasoni et al., 2004). Segmentation of the recording sessions into SWS, transition from awake/REM to SWS, transition from SWS to awake/REM, and awake/REM was performed as follows. Recordings were segmented into 1-min-long epochs. Those epochs, in which $\delta$ wave occurrence rate exceeded $10 /$ min, were considered as SWS. SWS epochs only separated by 1 min or less, were merged. The first minutes of SWS with the preceding awake, non-SWS epochs were labeled as "transition to SWS." Similarly, we identified the last minutes of SWS sessions and the first minutes of non-SWS, awake as "transition from SWS." Remaining epochs were labeled as "awake/REM."

To estimate the movement of the animals' electromyogram (EMG) was extracted from the intracranial signals by detecting the zero time-lag correlation coefficients $(r)$ between $300-$ and $600-\mathrm{Hz}$ filtered signals recorded at different sites. Pairwise Pearson correlations were calculated between pairs of channels that were minimally $400 \mu \mathrm{m}$ away from each other. The mean of all pairwise correlations measured in each 0.5-s bin was calculated and recorded as an EMG score (Schomburg et al., 2014). EMG signals were used to separate previously identified awake/ REM periods to awake and REM periods (REM periods correspond to low EMG, high $\theta / \delta$ ratio epochs) and to estimate animals' activity in awake periods.

Spindle detection was performed as follows. The LFP recorded in the somatosensory cortex was bandpass filtered $(10-20 \mathrm{~Hz})$ and Z-scored. Spindles corresponded to epochs where Z-score exceeded 2 for $>0.2 \mathrm{~s}$ and peaked at $>4$. Events separated by $<0.4 \mathrm{~s}$ were merged, and combined events lasting $>3 \mathrm{~s}$ were discarded (Maingret et al., 2016). The events induced by intracortical stimulation were classified manually. Continuous wavelet spectra and power spectra were calculated in MATLAB 
using Wavelet Toolbox and Chronux Toolbox (http:// chronux.org/), respectively.

The spike sorting was performed using Kilosort with its default settings (Pachitariu et al., 2016) and were manually refined in Phy (Rossant et al., 2016). Putative interneurons and pyramidal cells were discriminated based on their spike widths and autocorrelograms (Csicsvari et al., 1998; Mizuseki et al., 2009). For all individual units, spiking activity in a $[-88,+88]-m s$ window centered around the peak of SWDs were collected and firing rate histograms (0.8-ms time bin) were constructed. The firing rate histograms were Z-scored for each unit individually and smoothed with a Gaussian filter. Raster plots were constructed with all the smoothed Z-scored histograms of individual units.

\section{Histology}

At the end of the experiments, electrolytic lesions were made at the tip of the electrodes to verify their location. Animals were deeply anaesthetized with $1.5 \mathrm{~g} / \mathrm{kg}$ urethane (intraperitoneal) and transcardially perfused with saline followed by $4 \%$ paraformaldehyde and $0.2 \%$ picric acid in $0.1 \mathrm{M}$ phosphate buffer saline. After overnight postfixation, $50-\mu \mathrm{m}$-thick coronal sections were prepared with vibratome (VT1000S Vibratome, Leica) and stained with 4',6-diamidino-2-phenylindole dihydrochloride (DAPI; D8417, Sigma-Aldrich).

\section{Statistics}

All statistical analyses were performed in MATLAB (The MathWorks). No statistical methods were used to predetermine sample sizes, but the number of animal and recorded cells were similar to those employed in previous works. All tests were two-tailed. Non-parametric Wilcoxon signed-rank test, Kruskal-Wallis one-way analysis of variance, repeated measures analysis of variance, Kolmogorov-Smirnov test, Rayleigh's tests were used. To investigate spindle characteristics' change over time, from each analyzed recording sessions we randomly sampled 500 events of each animal and applied repeated measures ANOVA. To perform similar analysis on seizures, we randomly sampled 200 seizure events in each animal. Box plots represent median and 25th and 75th percentiles, and their whiskers the data range. Outlier values are not displayed on the figures, but they were always included in the statistical analysis. Linear regressions were performed using robust-linear regression and $p$ values for linear regressions tests the hypothesis that true correlation exists against a null-correlation. Modulation strength was calculated using mean resultant length of the phases (peaks of spike component of SWDs are at $180^{\circ}$ ) and units were considered to have phase locking to SWDs when $\alpha<0.05$ and $\kappa>0.1$ on Rayleigh's test of non-uniformity using the circular statistics toolbox provided by P. Berens (Berens, 2009).

\section{Results}

\section{Development of SWDs with maturation}

First, we determined how SWD incidence is influenced by maturation. We found that the total time in SWDs (expressed as the percentage of the 24-h-long recordings spent in SWDs) progressively increased during the observation period and saturated around the age of five months (Fig. $1 A, B$ ), which is in accordance with previous observations of Long-Evans rats reporting stable SWD parameters at the age of six to nine months (Shaw, 2007). SWD epochs were significantly longer in older animals (Fig. 1C) and mean length of SWDs showed the same saturation tendency as the total time spent in SWDs. SWD occurrence rate showed an early modest increase that remained steady (Fig. 1D). The temporal distribution of the SWDs also changed with age. In six-month-old animals, the median interevent interval was shorter (Fig. 1E). This result suggests that in well-developed absence epilepsy bursts of long seizures can be observed, which accounts for most of the time spent in seizures (Fig. 1F).

\section{Relationship between sleep spindles and SWDs}

As SWDs are often considered as aberrant sleep spindles of the thalamocortical circuitry (McCormick and Bal, 1997; Beenhakker and Huguenard, 2009), we investigated how the progression of SWD-generation impacts physiologic sleep spindles. We found that sleep spindles and SWDs' duration and amplitude distributions were overlapping at the age of three months, but they became distinct as the animals reached the age of six months (Fig. 2A,B). The peak spindle frequency did not change with age (data not shown, $n=15$ observations per animal in five animals, 500 spindles per each observations were compared; ANOVA factor: age $\left.F_{(14,34930)}=2.37 ; p=0.12\right)$, however, a gradual fall of spindle occurrence rate (Fig. $2 C$ ) and duration (Fig. 2D) was observed. The increase of total time spent in SWDs, indicative of the progression of the disease, showed strong inverse correlation with the decline of spindle incidence rate that raises the possibility of mutually exclusive mechanisms of spindle and SWD generation. (Fig. 2E).

\section{Effect of antiepileptic treatment on SWDs and sleep spindles}

Next, we investigated the effect of ETX, the drug of first choice in absence epilepsy, on sleep spindle occurrence (Fig. 3A). A single high dose injection of ETX $(100 \mathrm{mg} / \mathrm{kg}$ body weight; Richards et al., 2003) resulted in a prompt SWD suppression (0-120 min after injection, $3.35 \pm$ $3.76 \%$ of the vehicle injection), which was accompanied with a reduction in sleep spindle occurrence $(0-120 \mathrm{~min}$ after injection, $70.80 \pm 45.46 \%$ of the vehicle injection), too. Later, as the drug's plasma level decreased, ETX still had the potential to suppress SWD activity $(600-720 \mathrm{~min}$ after injection, $27.62 \pm 24.27 \%$ of the vehicle injection), but simultaneously sleep spindle occurrence was higher than the time-matched baseline activity of vehicle injection (600-720 min after injection, $156.72 \pm 60.18 \%$ of the vehicle injection). To further investigate, whether this increased spindle occurrence rate was only the mechanistic consequence of the increased SWD-free time of the animals, we disrupted spike-and-wave activity with on-demand transcranial electrical stimulation in three animals. We found, that although transcranial electrical stimulation 


\section{A}

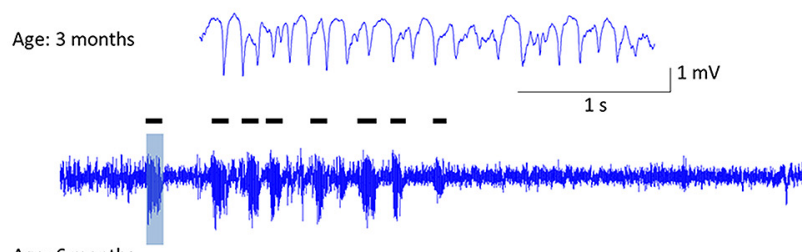

Age: 6 months
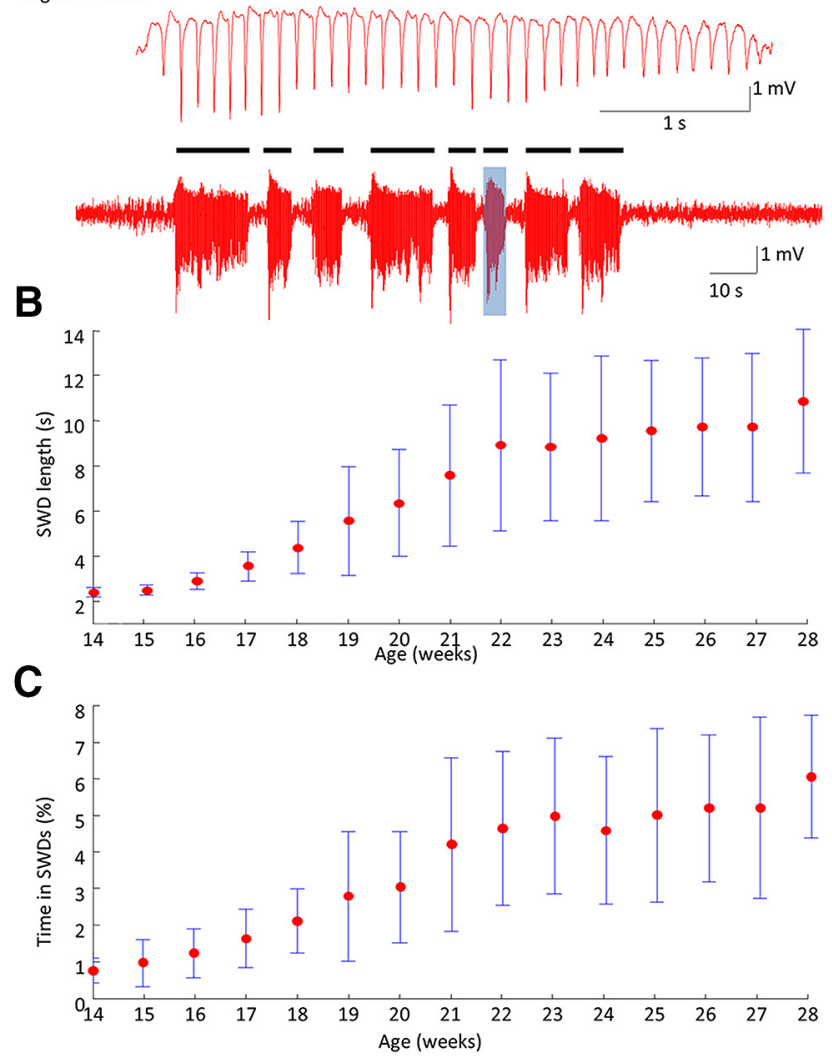

D

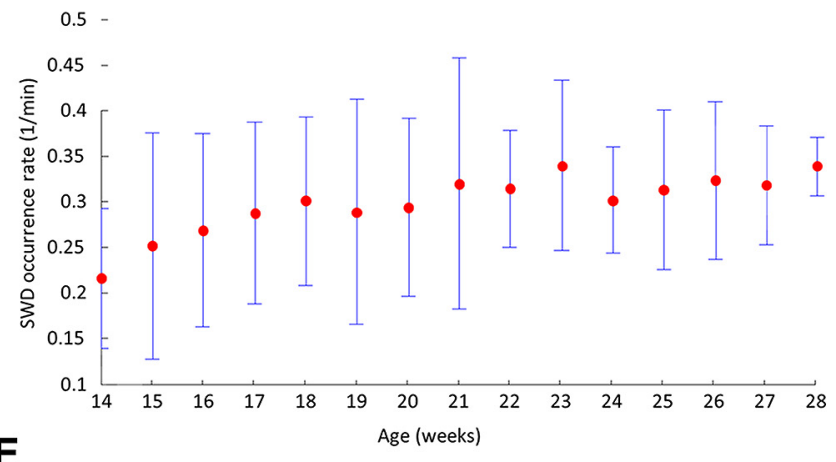

E

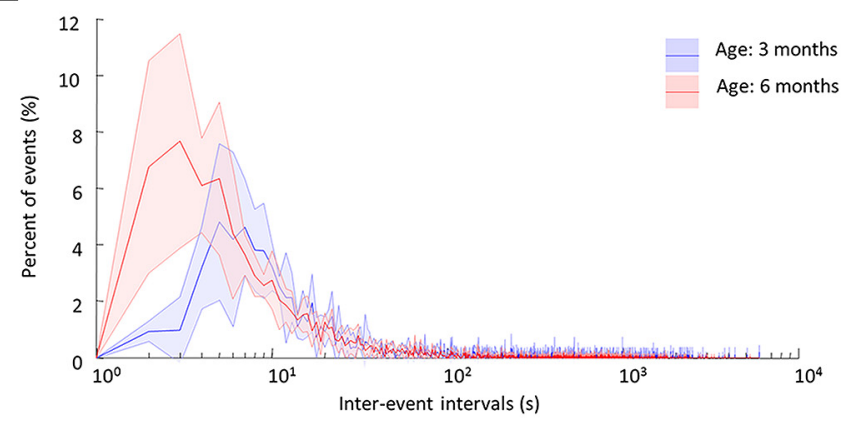

F

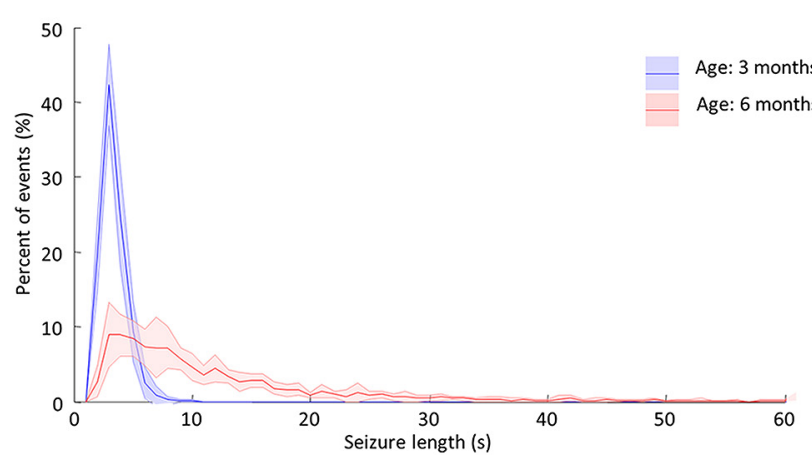

Figure 1. SWD frequency and duration increase with maturation. $\boldsymbol{A}$, Representative LFP traces of SWD activity at ages of 14 weeks (blue) and 28 weeks (red). Black ticks label individual seizures. Inlets show individual SWD epochs. Group data shows an increasing time spent in SWDs with age, expressed as the percentage of the total observation time $[(\boldsymbol{B}) n=15$ observations per animal in five animals; repeated measures ANOVA, factor: age $\left.F_{(14,56)}=13.60 ; p=0.0210\right)$, an increasing average SWD epoch duration; $(\boldsymbol{C}) n=$ 15 observations per animal in five animals; repeated measures ANOVA, factor: age $\left.F_{(14,56)}=18.51 ; p=0.0126\right)$ and increasing SWD rate; $(\boldsymbol{D}) n=15$ observations per animal in five animals; repeated measures ANOVA, factor: age, $\left.F_{(14,56)}=2.67 ; p=0.1773\right]$ over the observation period. Error bar represents \pm SD, data are sampled for $24 \mathrm{~h}$ during each week. Population data shows distribution of interevent intervals $(\boldsymbol{E})$ and the length of SWD epochs $(\boldsymbol{F})$ in the same animals $(n=5$ animals) at the age of three months (blue) and six months (red), shaded area represents \pm SD. Kolmogorov-Smirnov, interevent interval: $p<0.001$, distribution of seizures, $p<$ $0.001 ; * p \leq 0.05, * * p \leq 0.01, * * * p \leq 0.001$.

was effective in quickly terminating the SWDs $(n=3$ animals, as the total time spent in SWDs decreased), it did not decrease their incidence, and did not influence positively sleep quality by leaving sleep spindle occurrence rate unchanged (Fig. 3B).

\section{Brain state dependence of spontaneous SWDs}

Next, to further clarify the relationship of spontaneously emerging SWDs and physiologic thalamocortical oscillations (Beenhakker and Huguenard, 2009), we investigated how these patterns are generated around sleep. The incidence of spontaneous sleep spindles and SWDs with respect to the depth of the SWS (approximated by the density of $\delta$ waves, where higher density of $\delta$ waves corresponded to deeper SWS; Gervasoni et al., 2004; Lacroix et al., 2018) was different. Unlike sleep spindles, SWDs emerged at the transitions between wakefulness and sleep and their occurrence rate progressively fell with deepening of the SWS (Fig. 4A-C). Next, we analyzed the brain state dependence of SWD occurrence. Segmenting the recordings into SWS, transition to SWS, transition from SWS (2-min-long epochs with 1-min SWS and 1-min awake/REM period), and the rest as awake/REM sleep (Materials and Methods; Fig. 4D), we found that the SWD occurrence showed a substantial asymmetry regarding SWS (Fig. 4E). SWDs were likely to occur during transi- 
Sleep spindle and SWD characteristics (blue - spindle, red - SWD )

A
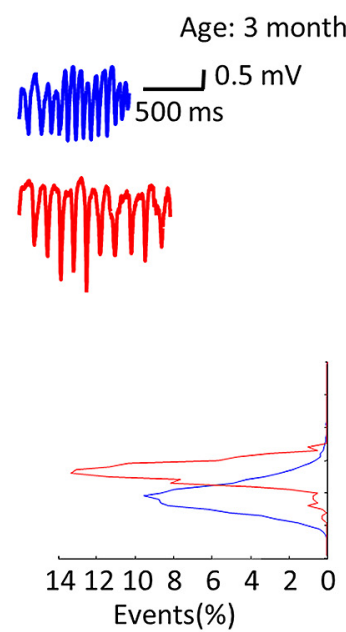

C

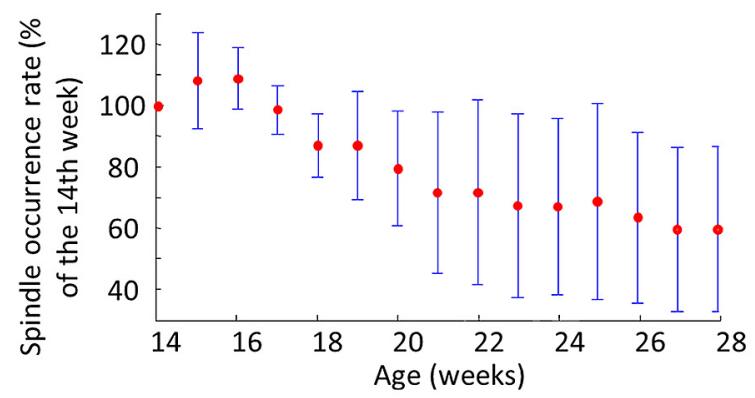

B
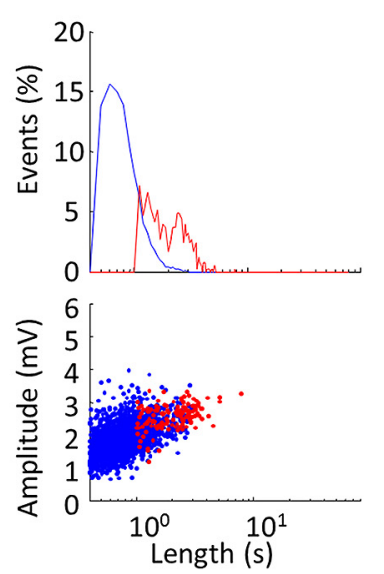

141210866420 Events (\%)

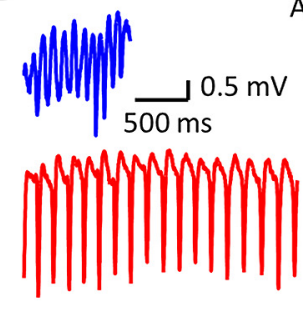

Age: 6 months
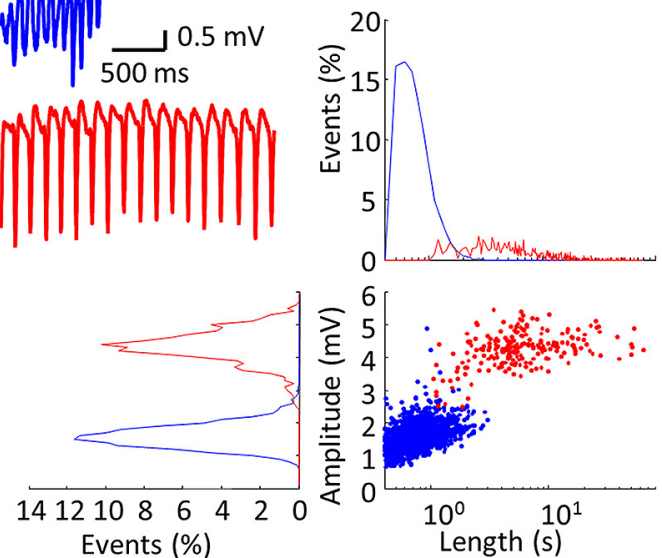

D

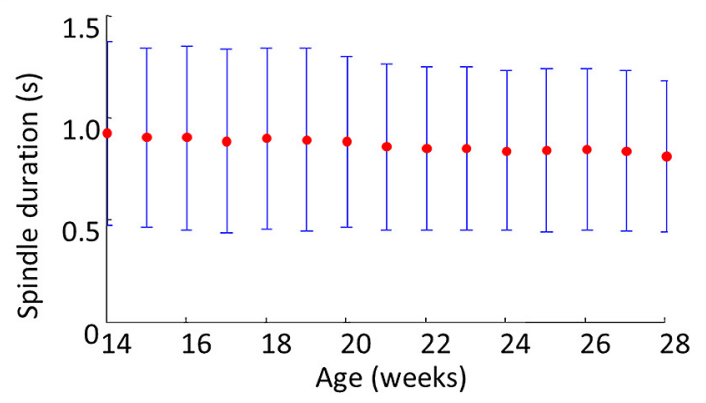

$\mathbf{E}$

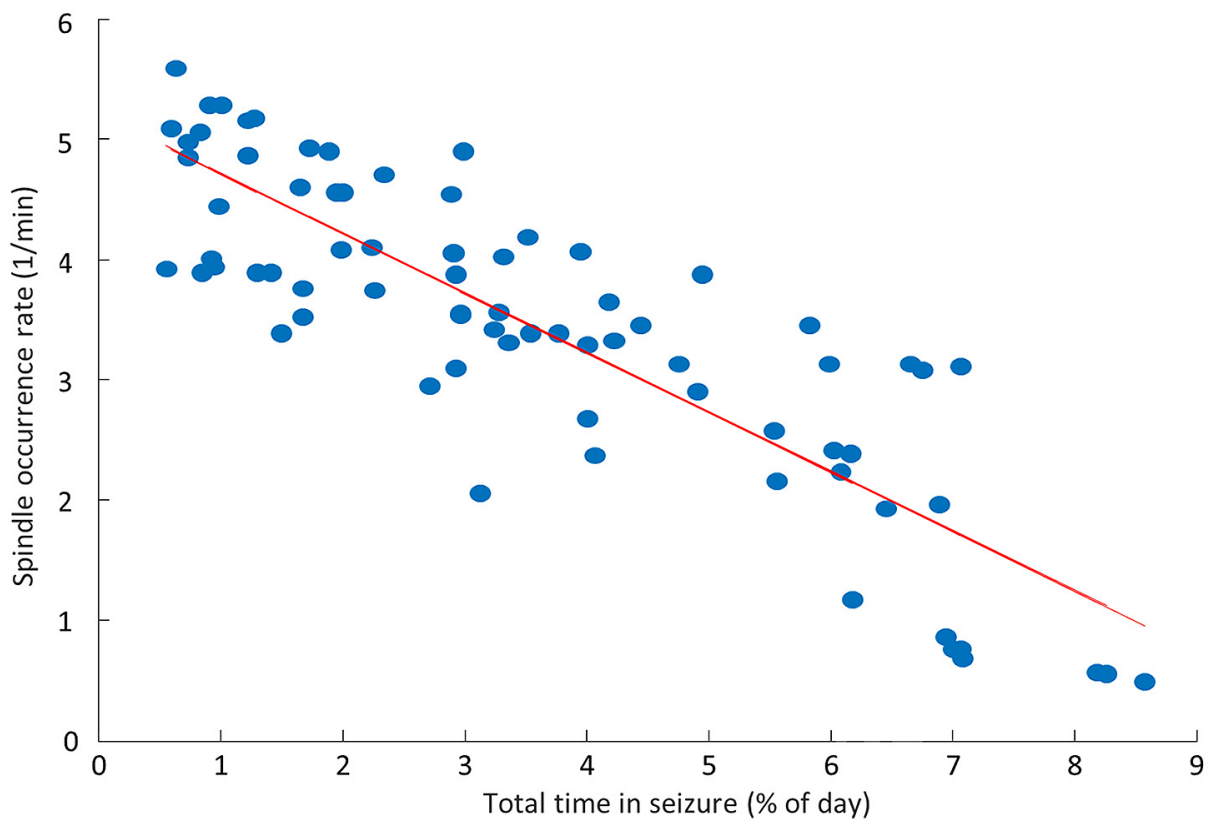

Figure 2. Relationship between sleep spindles and SWDs. $\boldsymbol{A}, \boldsymbol{B}$, Sleep spindle (blue) and seizure (red) characteristics in a representative animal at three and six months, with example sleep spindles and spike-and-wave episodes (upper left panel). $\boldsymbol{C}$, $\boldsymbol{D}$, Population data shows the decrease of sleep spindle duration $(n=15$ observations per animal in five animals, 500 randomly sampled spindles per each observation were compared; ANOVA factor: age $\left.F_{(14,34930)}=14.94 ; p<0.001\right)$ and sleep spindle incidence normalized to the first week of observation $\left(n=15\right.$ observations per animal in five animals; ANOVA $F_{(14,56)}=7.10 ; p=0.0562$, test was applied before normalization). $\boldsymbol{E}$, Regression of the total time spent in SWDs and sleep spindle occurrence rate on the population level $\left(n=15\right.$ observations per animal in five animals, $\left.R^{2}=0.73 ; p<0.001 ; t=-13.65\right)$, red line corresponds to best linear fit. 
A
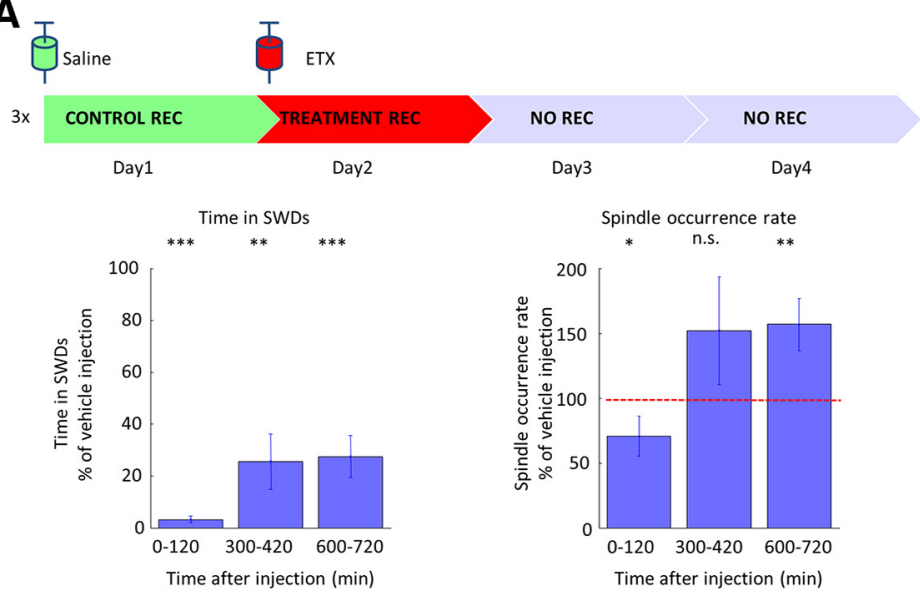

B

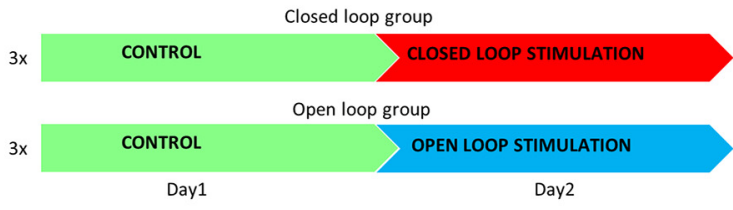

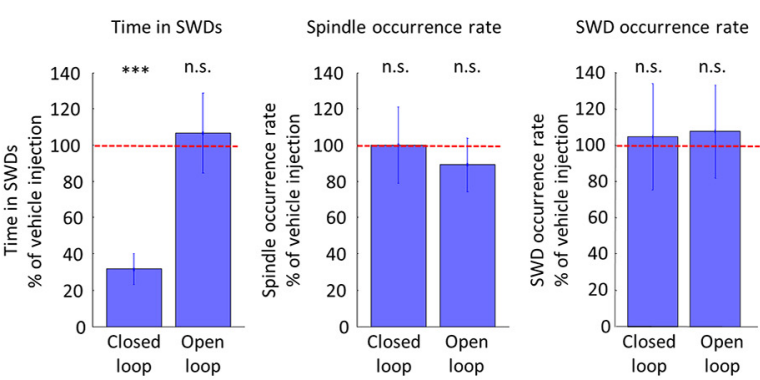

Figure 3. Effect of antiepileptic treatment on SWDs and sleep spindles. $\boldsymbol{A}$, The effect of ETX treatment on time spent in seizures (left) and on sleep spindle occurrence rate (right), expressed as the percentage of the control days' values ( $n=3$ control vs treatment session in three animals, Wilcoxon signed-rank test, control vs treatment). $\boldsymbol{B}$. The effect of closed loop and open loop transcranial electrical stimulation on time spent in seizures, on sleep spindle occurrence rate, and on the seizure occurrence rate $(n=3$ control vs treatment session in three animals, Wilcoxon signed-rank test, control vs treatment; data from Kozák and Berényi, 2017) expressed an the percentage of the control days' values. Error bars represent SEM; $* p \leq 0.05, * * p \leq 0.01, * * * p \leq 0.001$.

tions to SWS, but only few SWD epochs were observed during SWS and during transitions from SWS.

In absolute terms, the majority of the SWDs occurred in awake/REM state, but taking into account the time spent in different brain states, the transition to SWS found to be similarly susceptible to SWD generation (Fig. 4F). Next, based on derived EMG signals (see Materials and Methods), we removed from the previously identified Awake/ REM periods the REM sleep to investigate SWD susceptibility only in awake periods (REM corresponds to low EMG values with high $\theta / \delta$ ratio; Materials and Methods). and we found that SWDs were more likely to occur when the animals were not active (Fig. 4G,H), in accordance with previous findings (Marescaux et al., 1992; Steriade et al., 1993; Shaw, 2004; Smyk et al., 2019). Altogether, animals were susceptible to SWDs mainly in inactive resting wakefulness and when they were sleepy.

\section{Topographic differences and characteristics of SWDs}

Originally, absence epilepsy was considered as an instantaneously generalizing, global seizure type, but to date there is an increasing body of evidence suggesting that SWDs emerge locally from a cortical focus located in the somatosensory cortex (Meeren et al., 2002; Polack et al., 2007; Crunelli et al., 2011). Most currently available studies have investigated only the putative initiation zone of the ictal activity (Polack et al., 2007; Williams et al., 2016; Jarre et al., 2017), but have not clarified how other cortical areas are invaded or being surpassed by the SWDs.

Therefore, we systematically investigated SWDs emerging in the somatosensory cortex, on the neuronal activity of out-of-focus cortical areas. First, we compared the LFPs of the motor and the somatosensory cortices of the maturing rats during SWD epochs (Fig. 5A). The ictal LFP spectra of the different areas revealed that at the age of three months the somatosensory cortex already ex- pressed SWD oscillations (8.20 $\pm 2.10 \mathrm{~Hz}$; Fig. 5B), characteristic of SWDs. On the other hand, the motor cortex displayed a slower frequency oscillation $(7.36 \pm 2.29 \mathrm{~Hz})$, that gradually increased its frequency with maturation to reach the main SWD frequency $(8.23 \pm 0.78 \mathrm{~Hz}$; Fig. $5 C)$. Importantly, the bilateral somatosensory cortices showed higher coherence at the age of three months than the unilateral adjacent structures, but at the age of six months seizures became widely coherent with LFP activity locked to the SWD frequency (Fig. 5D). An interesting observation is that at the age of three months the characteristic frequency of the off-focus motor cortex often fell in the range of SWS during seizures in the somatosensory cortex and even $\delta$ waves were present (Fig. $5 E$ ).

\section{Firing dynamics of single units around SWDs}

Recent technical improvements made it possible to observe cortical network dynamics around seizures and an increasing body of evidence suggest that hypersynchronous SWDs are not necessarily accompanied by cellular level hyperactivity (Carney and Jackson, 2014; McCafferty et al., 2018; Meyer et al., 2018) To understand how off-focus cortical neurons contribute to generalizing SWD-patterns, we investigated how neuronal spiking of motor and prefrontal cortices were related to the spontaneously emerging SWDs (Fig. 6A). We compared the firing patterns during seizure emergence by looking at the firing rate of neurons (all neurons, putative excitatory pyramidal cells, putative inhibitory interneurons and inhibitory/ excitatory ratio) in a 2-s windows prior and following the seizure onset (Fig. 6B). During the preictal periods, the firing rate relationship of the putative excitatory cells (i.e., pyramidal cells) and the putative inhibitory cells (i.e., interneurons) did not change with maturation (firing rates of 118 vs 318 pyramidal cells, $Z=1.05, p=0.30 ; 77$ vs 113 interneurons' firing rate, $Z=1.69, p=0.09$, from juvenile and mature animals, respectively, Wilcoxon signed-rank test), suggesting no gross change in the overall inhibitory/ 
A

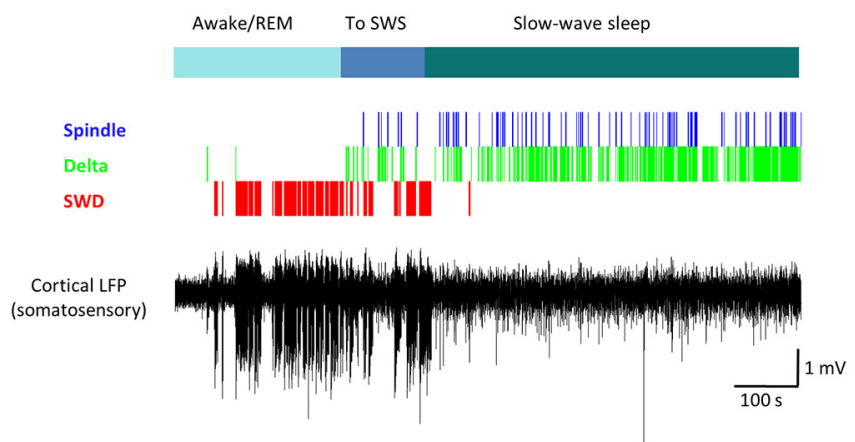

B

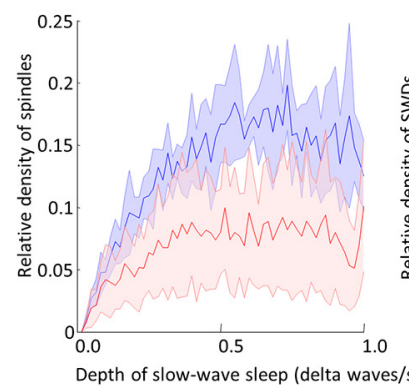

C
D

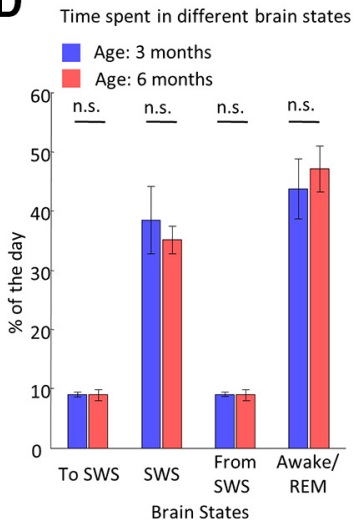

E
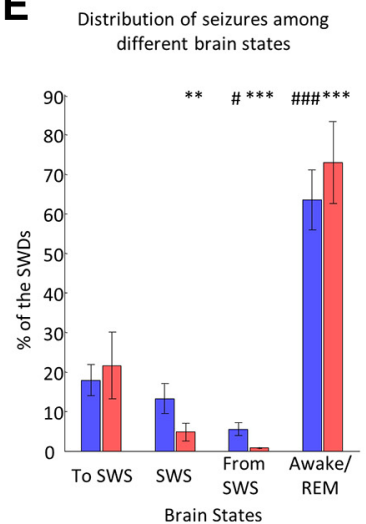

F

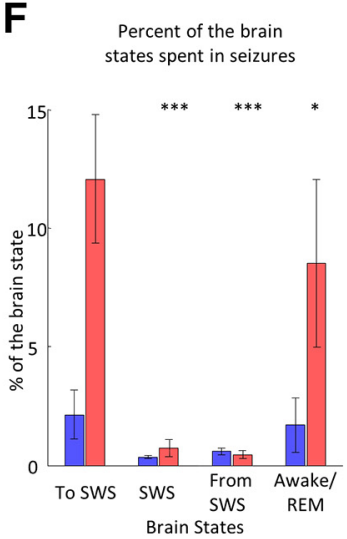

G

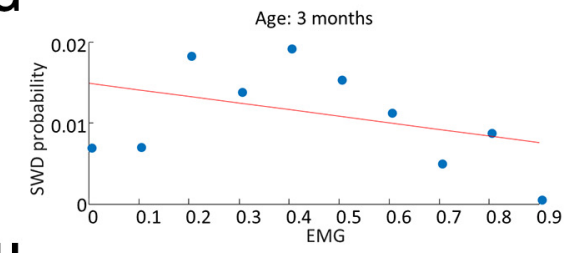

H

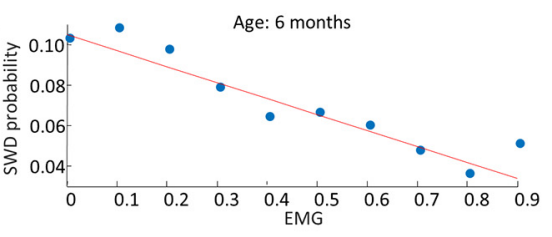

Figure 4. Brain state dependence of spontaneous SWDs. A, Representative wake-sleep transition of a mature rat. Black trace is the LFP of the somatosensory cortex, colored ticks highlight individual SWDs (red), $\delta$ waves (blue), and sleep spindles (green), classified brain states are showed on top in shades of blue. $\boldsymbol{B}, \boldsymbol{C}$, Relative occurrence of sleep spindles and SWDs as the function of $\delta$ wave density at three months (blue) and six months (red; Kolmogorov-Smirnov, sleep spindles: $p<0.001$, seizures: $p<0.05$ ). $\boldsymbol{D}$, Population data shows the time spent in different brain states at three months (blue) and six months (red) age in the same animals ( $n=5$ animals, two-way ANOVA, interaction between age and brain state, $\left.F_{(3,32)}=1.9 ; p=0.1492\right)$. E, Population data shows the distribution of SWD epochs among different brain states in the same animals $(n=5$ animals, two-way ANOVA, interaction between age and brain state, $F_{(3,32)}=4.78, p=0.0073$; post hoc Tukey-Kramer; vs three months To SWS: \#p $\leq 0.05$; \#\#\#p $\leq 0.001$; vs six months to SWS: $* * p \leq 0.01, * * * p \leq 0.001)$. $\boldsymbol{F}$, Population data shows the percentage of time spent in SWDs in different brain states $\left(n=5\right.$ animals, two-way ANOVA, interaction between age and brain state, $F_{(3,32)}=21.78, p<0.001$; post hoc Tukey-Kramer, vs six months to SWS: $* p \leq 0.05, * * * p \leq 0.001)$. $\mathbf{G}, \boldsymbol{H}$, Probability of SWD occurrence in awake animals as the function of preseizure normalized EMG signal at three and six months of age in the same animals. Red lines, best linear fit $(n=5$ animals, age: three months: $R^{2}=0.1554 ; p=0.2991 ; t=-1.1104$; age: six months: $R^{2}=0.8942 ; p<0.001 ; t=-7.8231$ ).

excitatory balance under non-ictal conditions. During the SWDs though, the firing rate of pyramidal cells did not change, while interneurons in mature animals with welldeveloped SWDs were less active compared to the preSWD baseline activity (in juveniles no difference was observed), causing the inhibitory/excitatory balance to shift toward excitation (Fig. 5C).

We next asked whether cells were entrained to seizure activity on a cycle-by-cycle basis or the LFP pattern of SWDs was more likely an emergent network property with loosely coupled unit activity, as it was reported in the thalamus (Buzsaki, 1991; Steriade and Contreras, 1995; McCafferty et al., 2018). We found that $53.3 \%$ (42.7\% of interneurons and $59.4 \%$ of pyramidal cells) of units in juvenile and $88 \%$ (89.6\% of interneurons and $87.3 \%$ of pyramidal cells) of mature animals' units showed phase locking to ongoing seizure activity (Fig. 6D-H). Interestingly, the preferred phase showed no cell-specific difference, but the pyramidal cells were significantly stronger coupled to SWDs (Fig. 6G,H). Furthermore, most cells were only firing in $\sim 25 \%$ of the total SWD cycles (Fig. 6/). It is possible, that since thalamocortical cells are not entrained in every cycle of the ictal activity (McCafferty et al., 2018), they do not provide thalamic feedback to their cortical counterparts on a cycle-to-cycle basis, and thus, most of the cortical cells are not constrained at each discharge even when an SWD is generalized.

\section{State-dependent seizure susceptibility}

Furthermore, to investigate SWD susceptibility, we tested how single-pulse intracortical electrical stimulation can influence brain activity. It is known that short singlepulse stimulation during NREM sleep can induce global $\delta$ waves, which are identical to the spontaneously emerging ones (Vyazovskiy et al., 2009; Maingret et al., 2016). Mechanistically, such electrical stimulation causes the simultaneous depolarization of some pyramidal cells, which in turn can entrain distant cortical regions into sleep oscillation via corticocortical connections (Vyazovskiy et al., 2009). Given that single-pulse stimulation has the 
A

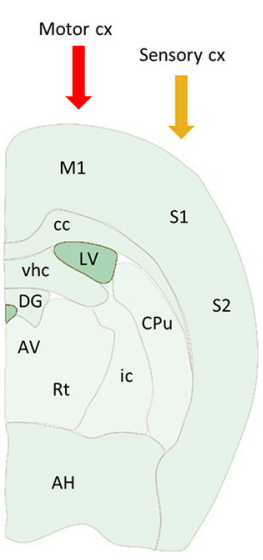

B
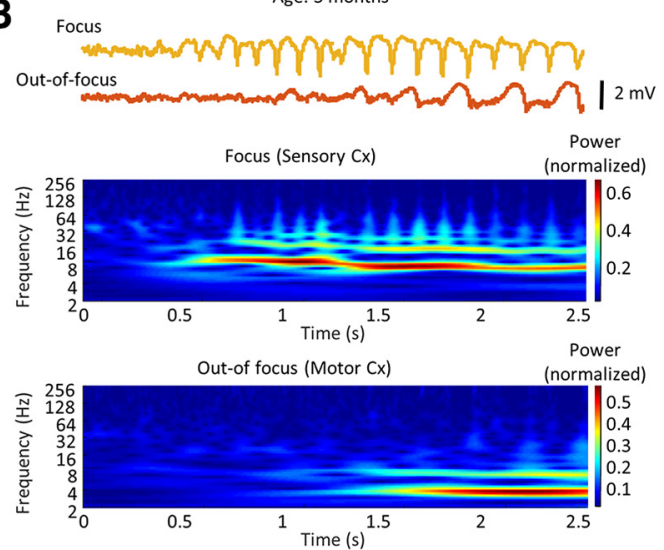

Age: 6 months

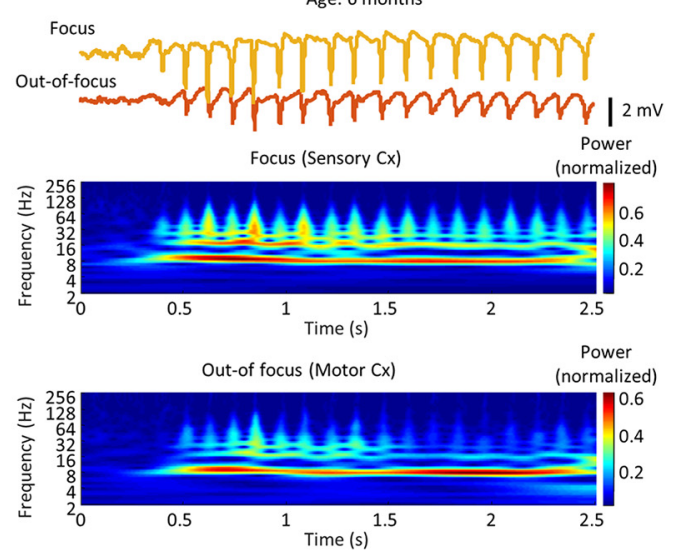

E
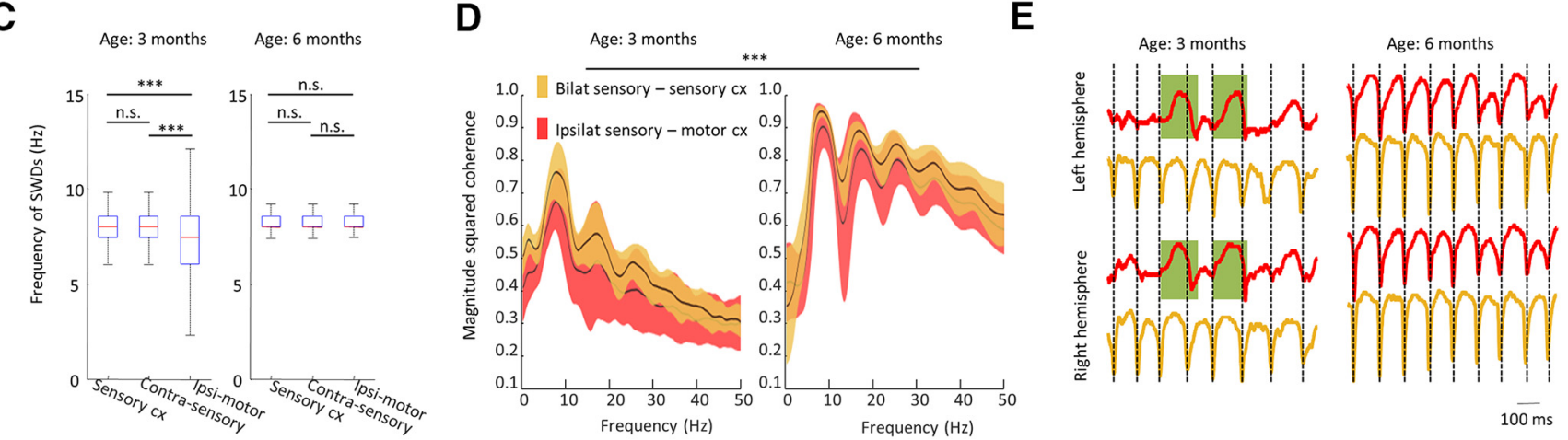

Figure 5. Topographic differences and characteristics of SWDs. $\boldsymbol{A}$, Typical arrangement of unilateral recording electrodes in the putative "initiation zone" (orange) and in the adjacent motor cortex (red). M1, primary motor cx; S1, primary somatosensory cx; S2, secondary somatosensory cx; CPu, caudate putamen; DG, dentate gyrus; AV, anteroventral thalamus; Rt, reticular thalamic nucleus; vhc, ventral hippocampal commissure; LV, lateral ventricle; cc, corpus callosum; ic, internal capsule; $\mathrm{AH}$, anterior hypothalamic area. $\boldsymbol{B}$, Representative LFP traces and corresponding wavelet spectrum of typical intraseizure activity of these cortical areas. $\boldsymbol{C}$, Population data shows mean frequency of spike and wave episodes in different cortical recording sites (ipsilateral and contralateral somatosensory cortices and ipsilateral motor cortex) at three and six months $(n=2$ observations per animal in five animals, 200 randomly sampled SWDs epochs per each observation were compared). $\boldsymbol{D}$, Population data shows increasing coherence of intraseizure LFP signals from three months to six months of age between different cortical recording sites in the same animals [bilateral somatosensory cortices (orange), ipsilateral somatosensory and motor cortices (red), $n=15$ observations per animal in five animals, two-way repeated measures ANOVA on peak coherence of intra-SWD LFP, factor: age $F_{(14,112)}=44.918 ; p<0.001$; factor: region (focus, off-focus), $\left.F_{(14,112)}=0.57076 ; p=0.4716\right]$. $E$, Representative LFP traces (orange, somatosensory cortex; red, motor cortex) show intraseizure dynamics at three and six months in the same animal. Dashed lines refer to the peak of SWDs, $\delta$ wave-like activity of motor cortices are highlighted; $* p \leq 0.05, * * p \leq 0.01, * * * p \leq 0.001$

potential to elicit global sleep patterns, we asked whether it is also sufficient to switch the brain into seizure activity. After determining the minimal intensity required to induce $\delta$ waves during SWS (Materials and Methods), we applied the same intensity stimulation during each brain state. Indeed, we were able to induce $\delta$ waves successfully as reflected by their characteristic LFP and unit-firing patterns (Fig. 7A). Interestingly, both juvenile and mature animals' somatosensory cortices could respond with spike-and-wave activity to the stimulation, although juvenile animals' ictal-like activity often remained local, but mature animals developed generalized seizures.

Next, we compared spiking activity around electrical stimuli to see how promptly the cortex can react to stimulation. to do that, we compared the firing rate of units (total, excitatory, inhibitory and inhibitory/excitatory) in 1-s windows prior and following stimuli. In juvenile animals, no change was observed in the investigated parameters, but in mature ones, total and excitatory firing rate increased after stimulation, therefore the inhibitory/excitatory balance shifted similarly to the spontaneously emerging seizures (Fig. 7B). Next, we determined the susceptibility of cortex for seizures in various brain states (Fig. 7C). In accordance with the spontaneously emerging seizures, stimulation during SWS were not able to induce seizure activity neither in juvenile nor in mature animals, but other brain states were susceptible for seizure induction. to determine what makes the cortex susceptible for seizure induction, we tested the correlation of seizure induction efficacy and the prestimulation power of characteristic oscillations (hippocampal $\theta$, cortical $\beta$ and $\delta$ ). We found that both hippocampal $\theta$ power and cortical $\delta$ power negatively correlated with seizure induction probability (Fig. $7 D, E, G, H$ ). The latter relationship is in line with the rare occurrence of spontaneous seizures and low yield of electrical triggering during NREM sleep. Interestingly, we 
A
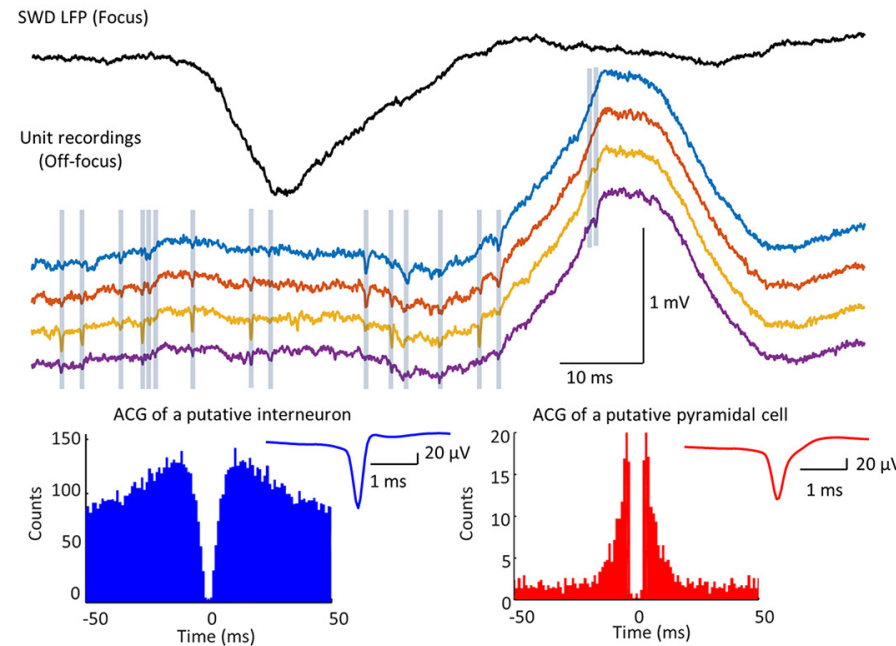

B

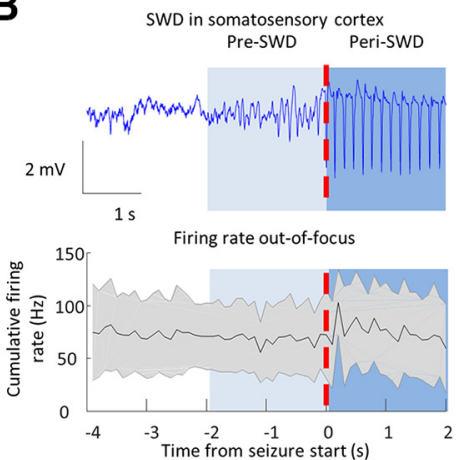

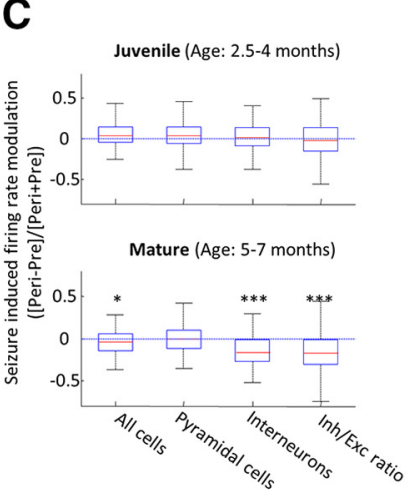

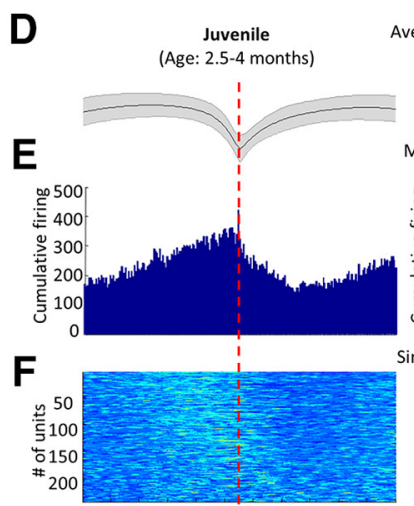

G
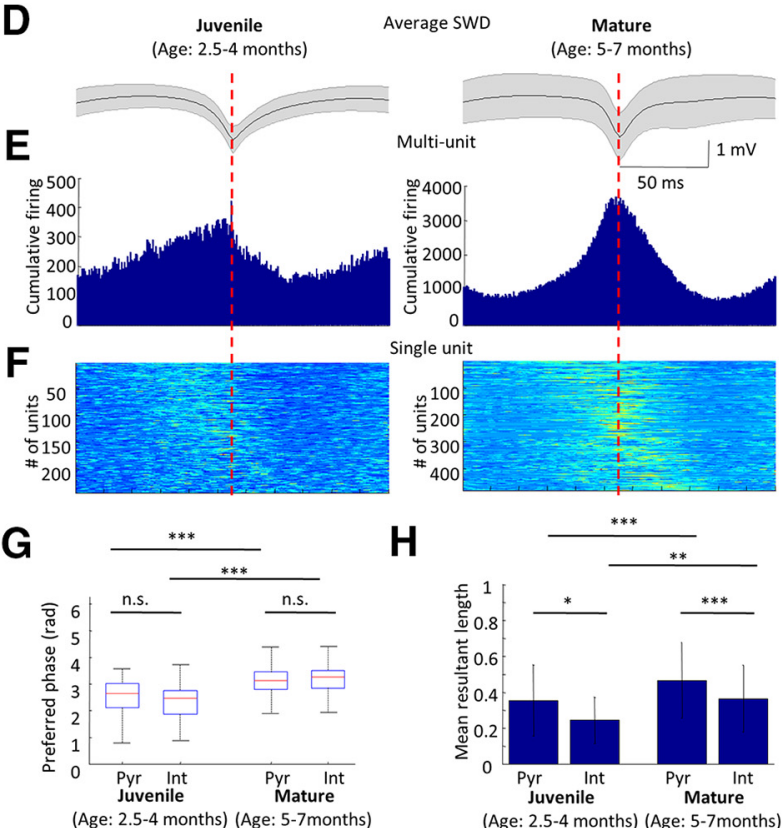

H

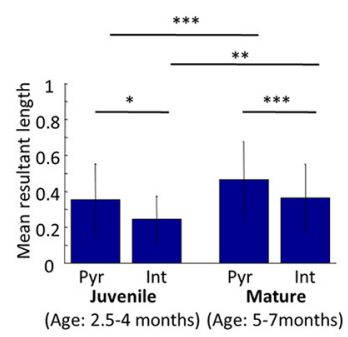

Unit involvement in ictal activity

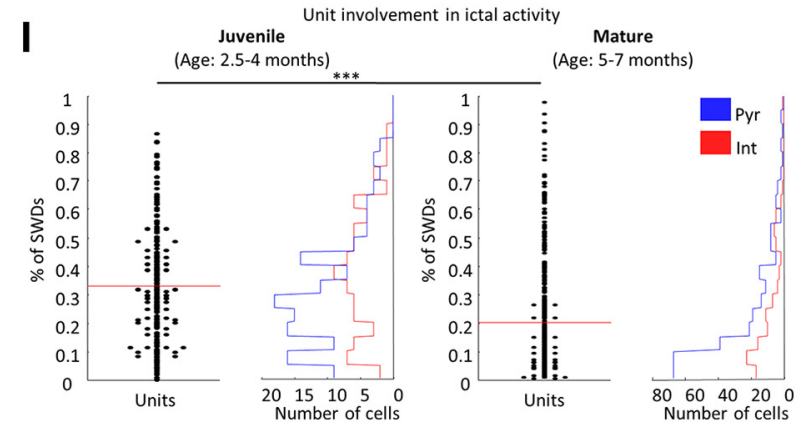

Figure 6. Firing dynamics of single units around SWDs. A, LFP trace of somatosensory cortex and total firing rate of motor cortex around seizure initiation. $\boldsymbol{B}$, Boxplot of firing rate ratios of units (all cells, pyramidal cells, interneurons and inhibitory/excitatory ratio) before and following the SWD onset ([Peri-Pre]/[Peri+Pre]; Wilcoxon signed-rank test, pre-SWD vs SWD, juvenile: $n=118$ pyramidal cells and $n=77$ interneurons, respectively, in 126 events of three animals; total $p=0.3136 ; Z=1.01$, excitatory $p=0.4542 ; Z=$ 0.75 , inhibitory $p=0.6986 ; Z=0.39$, inhibitory/excitatory $p=0.82485 ; Z=-0.22$; mature: $n=318$ pyramidal cells and 113 interneurons, respectively, in 215 events of four animals, total $p<0.05 ; Z=-2.26$, excitatory $p=0.9832 ; Z=0.02$, inhibitory $p<$ $0.001 ; Z=-3.98$, inhibitory/excitatory $p<0.001 ; Z=-5.93)$. $\boldsymbol{C}$, Average SWDs at different ages. $\boldsymbol{D}$, All unit and multiunit firing around SWDs. E, Unit activity around SWDs. F, Phase distribution of all spikes around SWDs. Peak of spike component corresponds to $180^{\circ}$. $\boldsymbol{G}, \boldsymbol{H}$, Phase preference and coupling strength of unit firing around SWDs for modulated interneurons and pyramidal cells [Kruskal-Wallis one-way analysis of variance, post hoc Wilcoxon signed-rank test, juvenile: $n=115 / 225$ (82/143 pyramidal cells, 33/82 interneurons) in three animals, mature: $n=351 / 408$ (252/293 pyramidal cells, 99/115 interneurons) in four animals, phase preference: $F_{(3,462)}=50.64 p<0.001$; mean resultant length: $\left.F_{(3,462)}=72.57 p<0.001\right]$. I, Unit involvement in ictal activity at each SWDs are plotted as percentage of SWDs in which they fire for each unit (left) and as the distribution of cell type-specific contribution [right, blue: pyramidal cell, red: interneuron; Wilcoxon signed-rank test, juvenile $(n=225$ units) vs mature $(n=408$ units) $Z=8.30$; $p<0.001]$. Red line represents mean; $* p \leq 0.05, * * p \leq 0.01, * * * p \leq 0.001$.

found a different seizure susceptibility regarding cortical $\beta$ band. In juveniles, $\beta$ power negatively correlated with seizure induction probability, but in contrast, it showed positive correlation in mature animals (Fig. $7 F, I)$.

Finally, we applied a rhythmic $8 \mathrm{~Hz}$ cortical stimulation to mimic the activity of a seizure focus in sleeping and in awake animals. We found that regardless the age, even rhythmic stimulation fails to induce sustainable seizures and immediately after stimulation withdrawal, cortex keeps on generating sleep-related patterns. On the contrary, in awake animals these stimulations easily induced seizures in both juvenile and mature animals (Fig. 7 J). This finding further supports the importance of brain state in seizure susceptibility.

\section{Discussion}

To our knowledge, this is the first study, which follows the evolution of thalamocortical rhythms in the same animals over the time course of three months in multiple cortical locations. We showed that maturation gradually shifts local ictal activity of the somatosensory cortex into global SWDs of the thalamocortical circuitry, which parallels the progressive disappearance of sleep spindles. Importantly, the sleep related changes seem to be revers- 
A
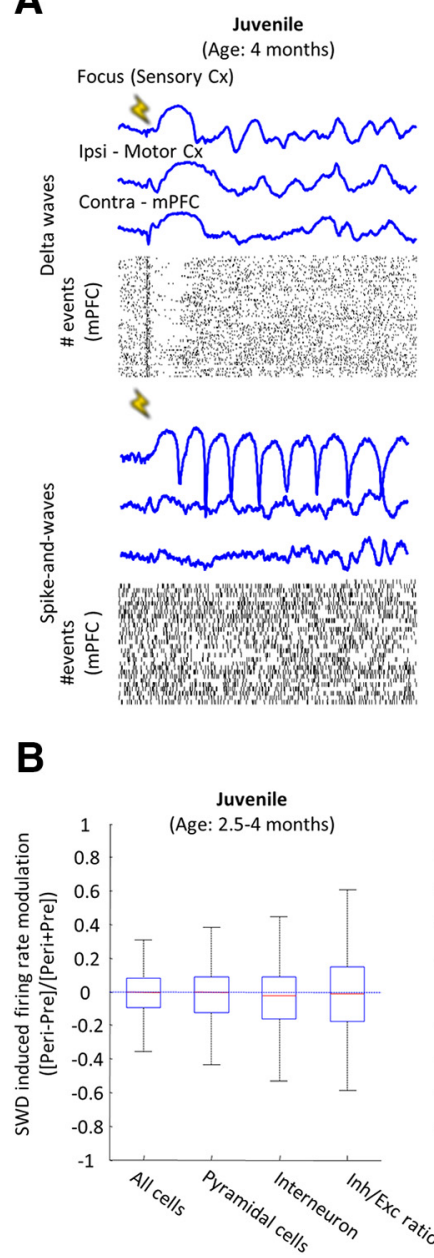
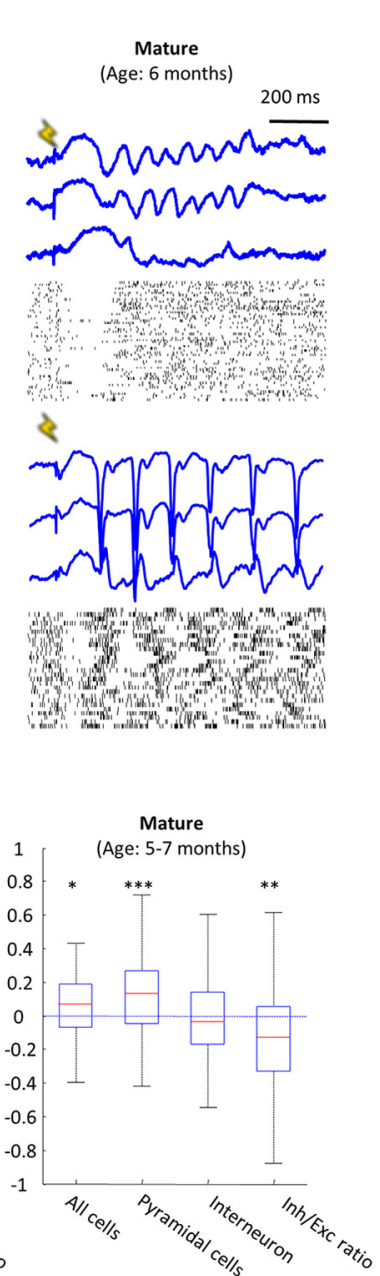

C

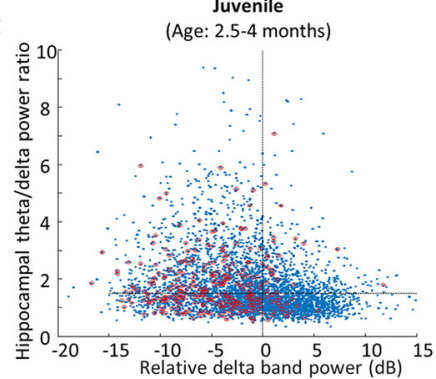

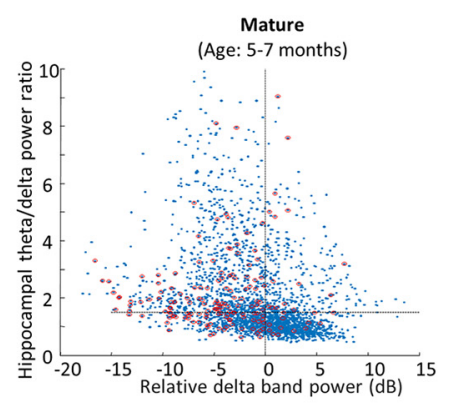
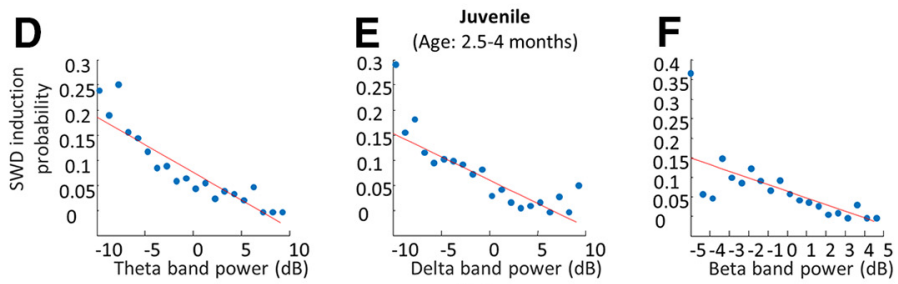

G
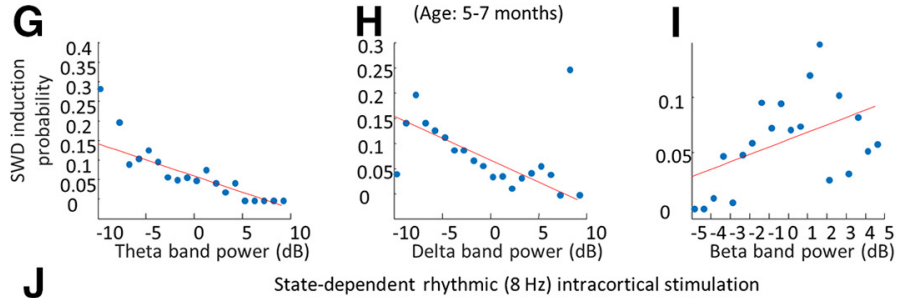

State-dependent rhythmic $(8 \mathrm{~Hz})$ intracortical stimulation Juvenile

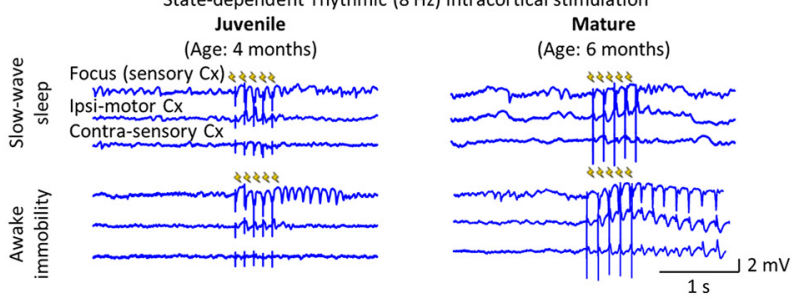

Figure 7. State-dependent seizure susceptibility. $\boldsymbol{A}$, Representative LFP traces (single event) of different cortical areas and raster plot of cortical units (mPFC) during stimulation trials, which successfully induced $\delta$ waves or spike and waves in a juvenile and a mature animal. $\boldsymbol{B}$, Boxplot of firing rate ratios of units (all cells, pyramidal cells, interneurons and inhibitory/excitatory ratio) around successful seizure induction ([Peri-Pre]/[Peri+Pre]; Wilcoxon signed-rank test, preseizure vs seizure, juvenile: $n=107$ pyramidal cell and $n=60$ interneurons, respectively, in three animals; total $p=0.8319 ; Z=-0.21$, excitatory $p=0.564 ; Z=-0.58$, inhibitory $p=0.5366$; $Z$ $=-0.62$, inhibitory/excitatory $p=0.74743 ; Z=-0.32$; mature: $n=317$ pyramidal cells and $n=117$ interneurons in four animals, total $p<0.05 ; Z=2.28$, excitatory $p<0.001 ; Z=3.48$, inhibitory $p=0.4969 ; Z=-0.68$, inhibitory/excitatory $p<0.01 ; Z=-3.01$ ). $\boldsymbol{C}$, Brain state dependence of single-pulse stimulations. Each blue dot represents a single trial as the function of cortical $\delta$ band power and hippocampal $\theta / \delta$ ratio. Dashed lines divide trials into four panels. Lower right panel corresponds to SWS. Red circles denote successful seizure inductions (juvenile: $n=10$ sessions in five animals, mature: $n=7$ sessions in four animals). $\boldsymbol{D}$, G, Population data shows probability of SWD induction as the function of prestimulus hippocampal $\theta$ band activity; $(\boldsymbol{E}, \boldsymbol{H})$ prestimulus cortical $\delta$ band activity and $(\boldsymbol{F}, \boldsymbol{I})$ prestimulus cortical $\beta$ band activity. Red lines: best linear fit (juvenile: $n=10$ sessions in five animals, mature: $n=$ 7 sessions in four animals; juvenile, $\theta: R^{2}=0.8425 ; p<0.001 ; t=-9.6547 ; \delta: R^{2}=0.7205 ; p<0.001 ; t=-8.8036 ; \beta: R^{2}=0.4724$; $p<0.001 ; t=-6.3559 ;$ mature, $\theta: R^{2}=0.6645 ; p<0.001 ; t=-6.4961 ; \delta: R^{2}=0.1416 ; p<0.001 ; t=-6.3944 ; \beta: R^{2}=0.2363$; $p<0.05 ; t=2.2121)$. $J$, Effect of $8-\mathrm{Hz}$ intracortical stimulation in juvenile and mature rats during NREM sleep and in awake state; $* p \leq 0.05, * * p \leq 0.01, * * * p \leq 0.001$.

ible by pharmacological therapy. Furthermore, we showed that even a single cortical depolarization burst is sufficient to kick a susceptible brain into seizure activity.

\section{Animal models of absence epilepsy}

Before discussing the implications of our findings for absence epilepsy, it is important to consider some issues related to the rodent models of absence epilepsy. Beside the inbred genetic models of absence epilepsy (WAG/Rij, GAERS; Coenen and Van Luijtelaar, 1987; Marescaux et al., 1992), it is known that Long-Evans (and other outbred strains, such as Wistar and Sprague Dawley) rats can express SWDs with similarity to absence seizures on the cellular (Polack and Charpier, 2006), behavioral (Semba et al., 1980) and pharmacological (Shaw, 2004) levels. Interestingly, the WAG/Rij, GAERS and Long-Evans strains show different age dependence of seizure development (Coenen and Van Luijtelaar, 1987; Jarre et al., 2017). The relative late appearance of absence seizures in Long-Evans rats made the continuous long-term recordings possible, as the growth of their skull is less during the 
observation period, thus they did not grow out the implants. Importantly, none of the rodent models of absence epilepsy can resemble the frequent spontaneous disappearance of seizures observed in humans (Crunelli and Leresche, 2002), thus there is an ongoing debate about how much the rodent SWDs can be used as a model to human absence epilepsy (Taylor et al., 2017). Most studies confirm the face validity, pharmacological predictive value and construct validity of rats in absence research (Depaulis and Charpier, 2017), thus, we consider the gradual development of the disease in the Long-Evans strain as an ideal temporal window to observe the sequence of network level changes at the cellular and network levels that may help to understand how seizures develop in human, too.

\section{Absence epilepsy and sleep spindles}

It is known, that absence epilepsy is very often accompanied with sleep disturbances in human (Myatchin and Lagae, 2007) and the progressive disappearance of sleep spindles might account for an impairment of learning abilities (Radek et al., 1994; Nolan et al., 2004), but the exact mechanisms are still elusive. We found that sleep architecture has changed during maturation. The NREM sleep became less spindle rich, as the spindle occurrence markedly decreased, but the peak frequency of the individual spindles did not change over the observation period. Altogether these findings raise the possibility of an impaired spindle initiation. Here, we showed that the parallel disappearance of sleep spindles and the emergence of spike and waves might be two joint consequences of the same underlying mechanism even if they affect different phases of sleep, as the treatment of absence epilepsy with ETX can increase the occurrence rate of sleep spindles in a dose-dependent manner. Similar results were found in human (Kellaway et al., 1990), although no systematic clinical study on antiepileptic drugs confirmed this effect of ETX treatment yet. The initial suppression of spindles is understandable as high dose of ETX might critically decrease the availability of T-type $\mathrm{Ca}^{2+}$ channels necessary for spindle initiation, but smaller plasma concentrations of ETX resulted in reduced seizure activity and high spindle occurrence. ETX exerts its pharmacological effect mainly via blocking T-type $\mathrm{Ca}^{2+}$ channels, although it has other targets as well and its seizure suppression effect is not confined to the thalamus (Manning et al., 2004). Thus, it has to be further investigated whether the frequent seizing is impairing the thalamocortical circuitry (Kozak, 2019) or a maturation-related cellular alteration may be in favor of seizure spread and causing spindle initiation difficulties in an already epileptic brain. Nevertheless, the restoration of sleep spindles by the antiepileptic drug of ETX suggests a network effect of channel deficiencies which causes spindle initiation difficulties in the epileptic brain, especially since the on-demand seizure control did not have similar positive effect on sleep spindles.

\section{Cortical single-cell activity during SWDs}

An interesting aspect of the results is that most cortical units, similarly to their thalamic counterparts (Buzsaki, 1991; McCafferty et al., 2018), are not firing during most of the spike and waves and altogether a distributed firing pattern of always-changing set of spike-and-wave entrained ensembles was observed during ictal activity with a decreased inhibitory/excitatory balance. Furthermore, it is possible that the symptoms of absence epilepsy, subjects being non-responsive for environmental stimuli during the seizures, are connected not only to the generalized synchronous activity of the minority of the units, but to the silence of the majority at the same time. Some studies showed intact cortical processing and partial consciousness during absence seizures in animals (Drinkenburg et al., 2003) and in human as well (Chipaux et al., 2013; Guo et al., 2016). The underlying reason for this might be that in each cycle, most cortical and thalamic units are not entrained to seizure activity, therefore the unaffected loops can still process incoming information from the external world and can explain why epileptic activity can be interrupted by unexpected sensory stimuli.

The overall decrease of firing of the inhibitory cells suggests that the silence of the units is not due to the powerful inhibition on the pyramidal cells but rather due to their weaker thalamocortical drive. On the other hand, our results show a higher inhibitory/excitatory balance in juvenile animals during ictal activity compared to mature ones, what raises the possibility of an active cortical inhibitory veto of seizure spread that disappears with age. Indeed, there is evidence that epileptic animals have an impairment of intracortical GABAergic inhibition (Luhmann et al., 1995; Crunelli et al., 2011). Importantly, our observations are in line with the results of Meyer et al. (2018), who showed using calcium imaging in the visual cortex of stargazer mice, that most inhibitory cells decrease their activity during absence seizures and most cells are not entrained to all of the SWD cycles. Additionally, a recent study investigating the activity of cortical pyramidal cells found that even in the seizure initiation zone pyramidal cells do not increase their firing rate during seizures (McCafferty et al., 2018).

An alternative mechanistic explanation of the decreased inhibitory activity is that while the bursts of the otherwise sparsely firing pyramidal cells during the spikes can counterbalance their decreased activity during the waves resulting in no gross change in their average firing rate, the already fast-firing interneurons cannot further increase their activity, and thus cannot compensate for their relative suppression during the wave phases. This speculation suggests that the decreased interneuron activity is a mere consequence of the altered rhythmicity, and thus, it may help to maintain the SW patterns but may not encounter for the increased susceptibility for seizure invasion of the given region. Further investigations are needed to clarify this question of mutual causality.

\section{Generalization of SWDs}

It is noteworthy, that while spindles are mainly global patterns already in the juvenile animals, the majority of 
both the spontaneous or induced ictal activity are local without generalization. This underscores that the hyperexcitable focus per se is not sufficient to drive generalized seizures, although the thalamocortical circuitry is already capable to generate generalized patterns. However, during maturation some yet undetermined changes happen which turn the whole brain to be susceptible for epileptic seizures. The hyperexcitability of the cortical focus develops earlier than the propensity of the adjacent areas to participate in the ictal activity, which suggests that seizure development is a multiple-step process.

A study showing that the unilateral inactivation of thalamus can disrupt epileptic seizures ipsilaterally presented similar cortical traces during contralateral seizure activity than we report here in juvenile animals (Buzsaki et al., 1988). As the cortical spread of ictal activity is hypothesized to happen through corticocortical connections, this highlights the importance of thalamocortical feedback on cortical units which frames them to fire synchronously to the seizure drive. Furthermore, the observed $\delta$ wave like activity of the motor cortex in response to the ongoing seizure activity of the somatosensory cortex in juvenile animals taken together with the very strong state dependence of seizure susceptibility raises the possibility that in juvenile animals the different cortical areas might be concomitantly in different microstates. Sleep studies reported that cortical areas can selectively go "off-line," a phenomenon known as local sleep (Vyazovskiy et al., 2011). Therefore, it is possible that the differential upstream determinants of cortical states locally can result in different response to incoming seizure drive, as one can develop a spike-and-wave and the other skips a cycle of the seizure with entering a $\delta$ wave. The existence of microstates reflects the independence of local thalamocortical loops to some extent and it may be possible that maturation increases the intrathalamic synchrony resulting in a more uniform response to cortical stimulation and generalization. A previous study already showed that ion channel loss in the thalamic reticular nucleus (TRN) cause the hypersynchrony of TRN neurons, leading to absence seizures (Makinson et al., 2017). Although it is important to mention that in such a multiple-step process a slight imbalanced shift to higher synchrony together with the already hyperexcitable cortex might probably be sufficient to initiate and maintain generalized seizures.

\section{State dependence of seizure susceptibility}

We showed here that spontaneous seizure occurrence correlates with changes in wakefulness. While the seizure probability is very high around transitions between wakefulness and light sleep, seizures are replaced by slow waves as sleep deepens and even rhythmic cortical activation fails to induce seizures in this state. It is known that arousal related brain states are controlled locally via the locus coeruleus noradrenergic system (Constantinople and Bruno, 2011). Furthermore, during transition to SWS the synchrony among TRN neurons is increased which is physiologically responsible for gating the sensory information to the cortex (Halassa et al., 2014). Alternatively, this also can be in favor of spreading highly synchronous activity of epileptic seizures (Makinson et al., 2017). It is possible that in maturing animals during wake-sleep transitions the thalamic synchrony is imbalanced in a way that the continuously decreasing arousal together with increasing TRN synchrony provides a window where the cortical excitability is still high enough to initiate a putative seizure and the TRN is already sufficiently synchronous to provide a sufficiently broad feedback to the cortex via the thalamocortical cells to frame seizure activity for the next cycle. We found that seizure susceptibility oppositely correlates with cortical $\beta$ oscillations in juvenile and in mature animals. The opposite correlation raises the possibility that these $\beta$ oscillations have different origin depending on the age of the animals. In juvenile animals it might represent physiologic $\beta$ oscillation, which is associated with attention and wakefulness, therefore negative correlation with seizure susceptibility is understandable. On the contrary, in mature animals, pathologic $\beta$ oscillation (Grandi et al., 2018) may emerge, which can contribute to seizure susceptibility. A recent study using optogenetic thalamic stimulation to induce seizures (Sorokin et al., 2016) described similar relationship between $\beta$ power and seizure induction probability as we found in mature rats. As they hypothesized, this cortical $\beta$ oscillation might synchronize and frame TRN firing to be pro-epileptic and making generalization of seizures possible.

We propose here that the development of absence epilepsy in polygenic models requires multiple steps, which very likely includes the development of a hyperexcitable cortical initiation zone and the occasional imbalance between thalamocortical synchrony and cortical excitability, which might be present in the form of pathologic $\beta$ oscillation. Altogether these multiple minor alterations might turn the thalamocortical dynamics to favor seizure spread in polygenic animal models and human subjects.

\section{References}

Bachmann K, Jahn D, Yang C, Schwartz J (1988) Ethosuximide disposition kinetics in rats. Xenobiotica 18:373-380.

Beenhakker MP, Huguenard JR (2009) Neurons that fire together also conspire together: is normal sleep circuitry hijacked to generate epilepsy? Neuron 62:612-632.

Berens P (2009) CircStat: a MATLAB toolbox for circular statistics. J Stat Softw 31:1-21.

Blumenfeld H (2005) Cellular and network mechanisms of spikewave seizures. Epilepsia 46[Suppl 9]:21-33.

Buzsaki G (1991) The thalamic clock: emergent network properties. Neuroscience 41:351-364.

Buzsaki G, Bickford RG, Ponomareff G, Thal LJ, Mandel R, Gage FH (1988) Nucleus basalis and thalamic control of neocortical activity in the freely moving rat. J Neurosci 8:4007-4026.

Carney PW, Jackson GD (2014) Insights into the mechanisms of absence seizure generation provided by EEG with functional MRI. Front Neurol 5:162.

Chipaux M, Vercueil L, Kaminska A, Mahon S, Charpier S (2013) Persistence of cortical sensory processing during absence seizures in human and an animal model: evidence from EEG and intracellular recordings. PLoS One 8:e58180.

Coenen AM, Van Luijtelaar EL (1987) The WAG/Rij rat model for absence epilepsy: age and sex factors. Epilepsy Res 1:297-301.

Constantinople CM, Bruno RM (2011) Effects and mechanisms of wakefulness on local cortical networks. Neuron 69:1061-1068.

Crunelli V, Leresche N (2002) Childhood absence epilepsy: genes, channels, neurons and networks. Nat Rev Neurosci 3:371-382. 
Crunelli V, Cope DW, Terry JR (2011) Transition to absence seizures and the role of GABA(A) receptors. Epilepsy Res 97:283-289.

Csicsvari J, Hirase H, Czurko A, Buzsáki G (1998) Reliability and state dependence of pyramidal cell-interneuron synapses in the hippocampus: an ensemble approach in the behaving rat. Neuron 21:179-189.

Depaulis A, Charpier S (2017) RE: loss of consciousness during absence seizures. [Electronic response to Taylor JA (2017) Voluntary control of epileptiform spike-wave discharges in awake rats.] J Neurosci 37:5861-5869.

Drinkenburg WH, Schuurmans ML, Coenen AM, Vossen JM, van Luijtelaar EL (2003) Ictal stimulus processing during spike-wave discharges in genetic epileptic rats. Behav Brain Res 143:141-146.

Gervasoni D, Lin SC, Ribeiro S, Soares ES, Pantoja J, Nicolelis MA (2004) Global forebrain dynamics predict rat behavioral states and their transitions. J Neurosci 24:11137-11147.

Grandi LC, Kaelin-Lang A, Orban G, Song W, Salvadè A, Stefani A, Di Giovanni G, Galati S (2018) Oscillatory activity in the cortex, motor thalamus and nucleus reticularis thalami in acute TTX and chronic 6-OHDA dopamine-depleted animals. Front Neurol 9:663.

Guo JN, Kim R, Chen Y, Negishi M, Jhun S, Weiss S, Ryu JH, Bai X, Xiao W, Feeney E, Rodriguez-Fernandez J, Mistry H, Crunelli V, Crowley MJ, Mayes LC, Constable RT, Blumenfeld H (2016) Impaired consciousness in patients with absence seizures investigated by functional MRI, EEG, and behavioural measures: a crosssectional study. Lancet Neurol 15:1336-1345.

Halassa MM, Chen Z, Wimmer RD, Brunetti PM, Zhao S, Zikopoulos B, Wang F, Brown EN, Wilson MA (2014) State-dependent architecture of thalamic reticular subnetworks. Cell 158:808-821.

Holmes MD, Brown M, Tucker DM (2004) Are "generalized" seizures truly generalized? Evidence of localized mesial frontal and frontopolar discharges in absence. Epilepsia 45:1568-1579.

Jallon P, Loiseau P, Loiseau J (2001) Newly diagnosed unprovoked epileptic seizures: presentation at diagnosis in CAROLE study. Coordination Active du Réseau Observatoire Longitudinal de I' Epilepsie. Epilepsia 42:464-475.

Jarre G, Altwegg-Boussac T, Williams MS, Studer F, Chipaux M, David O, Charpier S, Depaulis A, Mahon S, Guillemain I (2017) Building up absence seizures in the somatosensory cortex: from network to cellular epileptogenic processes. Cereb Cortex 27: 4607-4623.

Kellaway P Jr, J DF, Crawley JW (1990) The relationship between sleep spindles and spike-and-wave bursts in human epilepsy. In: Generalized epilepsy (Avoli M, Gloor P, Kostopoulos G, Naquet R, eds), pp 36-48. Boston: Birkhäuser.

Kozak G (2019) Absence epilepsy might build its own nest. J Physiol 597:1437-1438.

Kozák G, Berényi A (2017) Sustained efficacy of closed loop electrical stimulation for long-term treatment of absence epilepsy in rats. Sci Rep 7:6300.

Kozák G ,Földi T ,Berényi A (2018) Chronic transcranial electrical stimulation and intracortical recording in rats. J Vis Exp. Advance online publication. Retrieved May 11, 2018. doi: 10.3791/56669.

Lacroix MM, de Lavilléon G, Lefort J, El Kanbi K, Bagur S, Laventure S, Dauvilliers Y, Peyron C, Benchenane K (2018) Improved sleep scoring in mice reveals human-like stages. bioRxiv. Advance online publication. Retrieved December 7, 2018. doi: 10.1101/ 489005.

Luhmann HJ, Mittmann T, van Luijtelaar G, Heinemann U (1995) Impairment of intracortical GABAergic inhibition in a rat model of absence epilepsy. Epilepsy Res 22:43-51.

Maingret N, Girardeau G, Todorova R, Goutierre M, Zugaro M (2016) Hippocampo-cortical coupling mediates memory consolidation during sleep. Nat Neurosci 19:959-964.

Makinson CD, Tanaka BS, Sorokin JM, Wong JC, Christian CA, Goldin AL, Escayg A, Huguenard JR (2017) Regulation of thalamic and cortical network synchrony by Scn8a. Neuron 93:11651179.e6.
Manning JP, Richards DA, Leresche N, Crunelli V, Bowery NG (2004) Cortical-area specific block of genetically determined absence seizures by ethosuximide. Neuroscience 123:5-9.

Marescaux C, Vergnes M, Depaulis A (1992) Genetic absence epilepsy in rats from Strasbourg-a review. J Neural Transm Suppl 35:37-69.

McCafferty C, David F, Venzi M, Lo"rincz ML, Delicata F, Atherton Z, Recchia G, Orban G, Lambert RC, Di Giovanni G, Leresche N, Crunelli V (2018) Cortical drive and thalamic feed-forward inhibition control thalamic output synchrony during absence seizures. Nat Neurosci 21:744-756.

McCormick DA, Bal T (1997) Sleep and arousal: thalamocortical mechanisms. Annu Rev Neurosci 20:185-215.

Meeren HK, Pijn JP, Van Luijtelaar EL, Coenen AM, Lopes da Silva FH (2002) Cortical focus drives widespread corticothalamic networks during spontaneous absence seizures in rats. J Neurosci 22:1480-1495

Meeren H, van Luijtelaar G, Lopes da Silva F, Coenen A (2005) Evolving concepts on the pathophysiology of absence seizures: the cortical focus theory. Arch Neurol 62:371-376.

Meyer J, Maheshwari A, Noebels J, Smirnakis S (2018) Asynchronous suppression of visual cortex during absence seizures in stargazer mice. Nat Commun 9:1938.

Mizuseki K, Sirota A, Pastalkova E, Buzsáki G (2009) Theta oscillations provide temporal windows for local circuit computation in the entorhinal-hippocampal loop. Neuron 64:267-280.

Myatchin I, Lagae L (2007) Sleep spindle abnormalities in children with generalized spike-wave discharges. Pediatr Neurol 36:106111.

Nolan MA, Redoblado MA, Lah S, Sabaz M, Lawson JA, Cunningham AM, Bleasel AF, Bye AM (2004) Memory function in childhood epilepsy syndromes. J Paediatr Child Health 40:20-27.

Pachitariu M, Steinmetz NA, Kadir SN, Carandini M, Harris KD (2016) Fast and accurate spike sorting of high-channel count probes with KiloSort. In: Advances in neural information processing systems, pp 4448-4456. Vancouver: Neural Information Processing Systems Foundation.

Pinault D, Leresche N, Charpier S, Deniau JM, Marescaux C, Vergnes M, Crunelli V (1998) Intracellular recordings in thalamic neurones during spontaneous spike and wave discharges in rats with absence epilepsy. J Physiol 509:449-456.

Pinault D, Vergnes M, Marescaux C (2001) Medium-voltage 5-9-Hz oscillations give rise to spike-and-wave discharges in a genetic model of absence epilepsy: in vivo dual extracellular recording of thalamic relay and reticular neurons. Neuroscience 105:181-201.

Polack PO, Charpier S (2006) Intracellular activity of cortical and thalamic neurones during high-voltage rhythmic spike discharge in Long-Evans rats in vivo. J Physiol 571:461-476.

Polack PO, Guillemain I, Hu E, Deransart C, Depaulis A, Charpier S (2007) Deep layer somatosensory cortical neurons initiate spikeand-wave discharges in a genetic model of absence seizures. $J$ Neurosci 27:6590-6599.

Radek RJ, Curzon P, Decker MW (1994) Characterization of high voltage spindles and spatial memory in young, mature and aged rats. Brain Res Bull 33:183-188.

Richards DA, Manning JP, Barnes D, Rombola L, Bowery NG, Caccia S, Leresche N, Crunelli V (2003) Targeting thalamic nuclei is not sufficient for the full anti-absence action of ethosuximide in a rat model of absence epilepsy. Epilepsy Res 54:97-107.

Rossant C, Kadir SN, Goodman DFM, Schulman J, Hunter MLD, Saleem AB, Grosmark A, Belluscio M, Denfield GH, Ecker AS, Tolias AS, Solomon S, Buzsaki G, Carandini M, Harris KD (2016) Spike sorting for large, dense electrode arrays. Nat Neurosci 19:634-641.

Sanchez-Vives MV, McCormick DA (2000) Cellular and network mechanisms of rhythmic recurrent activity in neocortex. Nat Neurosci 3:1027-1034.

Schomburg EW, Fernández-Ruiz A, Mizuseki K, Berényi A, Anastassiou CA, Koch C, Buzsáki G (2014) Theta phase segregation of 
input-specific gamma patterns in entorhinal-hippocampal networks. Neuron 84:470-485.

Semba K, Szechtman H, Komisaruk BR (1980) Synchrony among rhythmical facial tremor, neocortical 'alpha' waves, and thalamic non-sensory neuronal bursts in intact awake rats. Brain Res 195: 281-298.

Shaw FZ (2004) Is spontaneous high-voltage rhythmic spike discharge in Long Evans rats an absence-like seizure activity? J Neurophysiol 91:63-77.

Shaw FZ (2007) 7-12 Hz high-voltage rhythmic spike discharges in rats evaluated by antiepileptic drugs and flicker stimulation. $J$ Neurophysiol 97:238-247.

Sirota A, Montgomery S, Fujisawa S, Isomura Y, Zugaro M, Buzsáki G (2008) Entrainment of neocortical neurons and gamma oscillations by the hippocampal theta rhythm. Neuron 60:683-697.

Smyk MK, Sysoev IV, Sysoeva MV, van Luijtelaar G, Drinkenburg WH (2019) Can absence seizures be predicted by vigilance states? Advanced analysis of sleep-wake states and spike-wave discharges' occurrence in rats. Epilepsy Behav 96:200-209.

Sorokin JM, Paz JT, Huguenard JR (2016) Absence seizure susceptibility correlates with pre-ictal $\beta$ oscillations. J Physiol Paris 110: 372-381.

Steriade M, Domich L, Oakson G (1986) Reticularis thalami neurons revisited: activity changes during shifts in states of vigilance. $J$ Neurosci 6:68-81.

Steriade M, Contreras D (1995) Relations between cortical and thalamic cellular events during transition from sleep patterns to paroxysmal activity. J Neurosci 15:623-642.
Steriade M, McCormick DA, Sejnowski TJ (1993) Thalamocortical oscillations in the sleeping and aroused brain. Science 262: 679-685.

Studer F, Laghouati E, Jarre G, David O, Pouyatos B, Depaulis A (2019) Sensory coding is impaired in rat absence epilepsy. J Physiol 597:951-966.

Taylor JA, Rodgers KM, Bercum FM, Booth CJ, Dudek FE, Barth DS (2017) Voluntary control of epileptiform spike-wave discharges in awake rats. J Neurosci 37:5861-5869.

Timofeev I, Steriade M (2004) Neocortical seizures: initiation, development and cessation. Neuroscience 123:299-336.

Vandecasteele M, M S, Royer S, Belluscio M, Berenyi A, Diba K, Fujisawa S, Grosmark A, Mao D, Mizuseki K, Patel J, Stark E, Sullivan D, Watson B, Buzsaki G (2012) Large-scale recording of neurons by movable silicon probes in behaving rodents. J Vis Exp. Advance online publication. Retrieved March 4, 2012. doi: 10.3791/3568.

Vyazovskiy VV, Faraguna U, Cirelli C, Tononi G (2009) Triggering slow waves during NREM sleep in the rat by intracortical electrical stimulation: effects of sleep/wake history and background activity. J Neurophysiol 101:1921-1931.

Vyazovskiy VV, Olcese U, Hanlon EC, Nir Y, Cirelli C, Tononi G (2011) Local sleep in awake rats. Nature 472:443-447.

Williams MS, Altwegg-Boussac T, Chavez M, Lecas S, Mahon S, Charpier S (2016) Integrative properties and transfer function of cortical neurons initiating absence seizures in a rat genetic model. J Physiol 594:6733-6751. 